Acta Crystallographica Section B

Structural

Science

ISSN 0108-7681

\section{Francesca P. A. Fabbiani,* David R. Allan, Simon Parsons and Colin R. Pulham}

School of Chemistry and Centre for Science at Extreme Conditions, The University of Edinburgh, King's Buildings, West Mains Road, Edinburgh EH9 3J, Scotland

Correspondence e-mail:

f.p.a.fabbiani@ed.ac.uk
(C) 2006 International Union of Crystallography Printed in Great Britain - all rights reserved

\title{
Exploration of the high-pressure behaviour of polycyclic aromatic hydrocarbons: naphthalene, phenanthrene and pyrene
}

The structural response of three members of the family of polycyclic aromatic hydrocarbons (PAHs) to high-pressure recrystallization from dichloromethane solutions is presented. Recrystallization of naphthalene in the 0.2-0.6 GPa pressure range does not result in the formation of a new polymorph. Furthermore, direct compression of a single crystal to $2.1 \mathrm{GPa}$ does not result in a phase transition. A density decrease of $18.2 \%$ over the $0.0-2.1 \mathrm{GPa}$ pressure range is observed and the principal effect of pressure is to 'tighten' the herringbone structural motif and decrease the size of void regions. A new polymorph of pyrene, form III, has been crystallized at 0.3 and at $0.5 \mathrm{GPa}$. Structural investigation of this new polymorph by means of topological analysis and comparison of Hirshfeld surfaces and fingerprint plots shows that intermolecular interactions are substantially different from those found in the ambient-pressure structures, and do not fit a previously established packing model for PAHs. Similar discrepancies are found for the high-pressure polymorph of phenanthrene, which is here re-investigated in greater detail. The structures of these high-pressure polymorphs are dominated by $\pi \cdots \pi$ stacking with a limited contribution from $\mathrm{C}-\mathrm{H} \cdots \pi$ (peripheral) interactions. It is perhaps not surprising that highpressure polymorphs deviate from a model that has been devised for ambient-pressure structures, and this may be a direct consequence of the ability of pressure to modify and combine intermolecular interactions in ways that are not usually found at ambient pressure. This is achieved by modifying the relative orientations of molecules and by encouraging the formation of denser structures in which molecules pack together more efficiently.

\section{Introduction}

The study of the structural response to external action, such as increasing pressure or decreasing temperature, is an effective way of probing intermolecular interactions. Pressure, with temperature, is a means of inducing phase transitions and the application of pressure can therefore be used to induce the formation of new polymorphs. Comparative studies of polymorphs can provide a better understanding of intermolecular interactions and of the factors that influence phase transitions between them. The effects of pressure on molecular crystals have been recently reviewed in the literature by Hemley \& Dera (2000) and by Boldyreva (2004a).

To date, most success in obtaining new polymorphs of organic compounds at high pressure has been achieved with compounds that are liquids at or near ambient temperature and pressure. Attempts to induce polymorphism in more complex compounds that have high melting points have been much less successful: this is because thermal decomposition of
Received 30 April 2006 Accepted 11 July 2006 
the compound usually occurs long before the melting temperature is reached. This is exacerbated by the increase in melting point with increasing pressure. An alternative method is the direct compression of the material (either as a singlecrystal or as a powder) contained in a diamond-anvil cell (DAC). Whilst this method can be effective for inducing phase transitions in compounds containing small molecules that have some conformational flexibility, experience has shown that the method is less effective for solids containing larger, more complex molecules. Even though the application of pressure to larger organic molecules may thermodynamically favour the adoption of a new polymorphic form, there is often a substantial kinetic barrier to be overcome before the molecules can rearrange. This observation appears to be supported by the recent results of Boldyreva et al. (2002), who have studied the effect of pressure on paracetamol and have shown that although it is possible to induce conversion of the monoclinic form to the orthorhombic form, the conversion is only partial, poorly reproducible and is only observed if a powder sample is used.

To overcome the difficulties mentioned above, a different technique has been developed instead (Fabbiani et al., 2003, 2004; Fabbiani, Allan, Parsons \& Pulham, 2005; Fabbiani, Allan, Marshall, Parsons, Pulham \& Smith, 2005). This involves growing single crystals from solution at high pressure. The technique removes the need for excessively high temperatures, overcomes the barrier to molecular rearrangement (lattice energy is overcome by solvation energy) and also provides an opportunity to study high-pressure crystallization from different solvent systems. This concept has been widely applied in the hydrothermal growth of inorganic materials such as quartz and other minerals (Hervey \& Foize, 2001), but until recently the technique has not been applied to organic compounds contained in a DAC.

\section{Polycyclic aromatic hydrocarbons}

The structural response of three members of the family of polycyclic aromatic hydrocarbons (PAHs) to recrystallization under conditions of high pressure is discussed in this paper. These are naphthalene, phenanthrene and pyrene (Fig. 1).

PAHs are natural constituents of coal tar and are present in gasoline and diesel fuels (Harvey, 1991). Naphthalene and phenanthrene are used as intermediates in the production of a variety of industrial products. Pyrene is a compound of high toxicity and a suspected human carcinogen. All three compounds are readily obtained by recrystallization from solutions of organic solvents (typically methanol or dichloro-

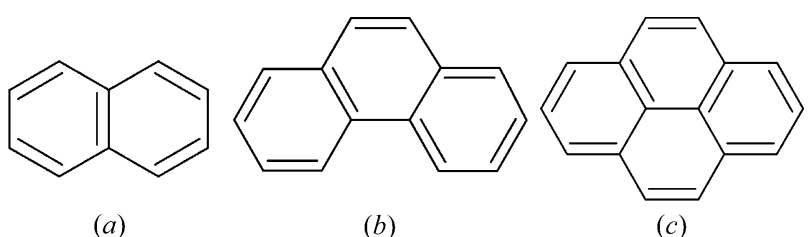

Figure 1

Molecular structure of $(a)$ naphthalene, $(b)$ phenanthrene and $(c)$ pyrene. methane) at ambient temperature and pressure and all compounds have been studied to some extent both at low temperatures and at high pressures.

\subsection{Naphthalene}

The first published crystal structure of naphthalene is that of Abrahams et al. (1949), who reported that naphthalene crystallizes at ambient pressure in the monoclinic crystal system, space group $P 2_{1} / a$ with the molecule residing on an inversion centre to give half a crystallographically independent molecule in the asymmetric unit. The diffraction data of Abrahams et al. (1949) were later refined in detail by Cruickshank (1957), who also analysed thermal motion. Thermal motion was also investigated by Ponomarev et al. (1976), Brock \& Dunitz (1982) and more recently by Oddershede \& Larsen (2004) in a series of single-crystal experiments at multiple temperatures. Perdeuteronaphthalene $\left(\mathrm{C}_{10} \mathrm{D}_{8}\right)$ has also been studied by neutron powder diffraction by Pawley \& Yeats (1969) and later by Natkaniec et al. (1983).

High-pressure studies on naphthalene have attracted considerable and varied interest: Goworek et al. (2004) found that the lifetime of ortho-positronium in solid naphthalene at $0.1 \mathrm{GPa}$ shortens as a result of diminishing free space in which it can be trapped, whilst Davydov et al. (1996) studied the kinetics of carbonization and graphitization of naphthalene in the $0.5-2.5 \mathrm{GPa}$ pressure range.

Bridgman (1938) found using volumetric apparatus an 'unmistakable transition' in naphthalene at ca 3.0 GPa. The volume change associated with this transition was very small and the transition was sluggish and showed hysteresis. The transition was subsequently detected at $c a 2.6 \mathrm{GPa}$ in shearing experiments (Gonikberg et al., 1966). Jones \& Nicol (1968) suggested that changes observed in the fluorescence spectrum of a single crystal of naphthalene below 4.0 GPa may have arisen from a phase transition. Block et al. (1970) observed the transformation by optical microscopy in a DAC at $c a 3.0 \mathrm{GPa}$, but Vaidya \& Kennedy (1971) failed to detect it using volumetric measurements to $4.0 \mathrm{GPa}$. IR studies in a DAC (Hamann, 1978) indicated that the transition is first detected at an average pressure of $2.0 \mathrm{GPa}$, although traces of the original spectrum were still present at $4.5 \mathrm{GPa}$, in line with Bridgman's observation of a sluggish transition.

In contrast to some spectroscopic evidence, X-ray powder diffraction experiments at room temperature up to $0.51 \mathrm{GPa}$ (Alt \& Kalus, 1982) gave no indication of a phase transition. These results are in agreement with Raman studies indicating that the naphthalene crystal undergoes no structural phase transition, at least up to $3.6 \mathrm{GPa}$ (Nicol et al., 1975) and $450 \mathrm{~K}$. A comparison of the literature data, which appear to be contradictory, is particularly difficult since in some cases no pressure-transmitting medium was employed, in others it is not given and in the remaining cases different fluids were used.

The results of these studies were intriguing and suggested that the compound is an ideal candidate for study by highpressure recrystallization from solution. In addition, naphthalene is one of the extensively studied compounds that have 
shown no evidence of polymorphic behaviour at ambient pressure, 'even though they have been crystallized and handled for many years under a far-ranging variety of conditions' (Dunitz \& Bernstein, 1995; the two other most cited examples are sucrose and Pigment Red 179) and stimulated some curiosity as to whether the new high-pressure technique could challenge this statement.

\subsection{Phenanthrene}

The structure of the ambient-temperature phase, monoclinic, space group $P 2_{1}$ [form (II)], was first determined by Basak (1950) and later by Kay et al. (1971) and Petříček et al. (1990).

Phenanthrene undergoes a reversible phase transition at 339-344 K that was first discovered by Ueberreiter \& Orthmann (1950) and subsequently studied by Kroupa et al. (1988). The structure of the high-temperature phase, monoclinic, space group $P 2_{1} / a$, here denoted as form (I), was elucidated by Petříček et al. (1990), who showed that the transition gives an orientationally disordered phase with approximately equal occupancies (55:45) of the two orientations. The hightemperature phase can be quenched by rapid cooling to $248 \mathrm{~K}$.

Fluorescence spectra of phenanthrene measured as a function of pressure (Jones \& Nicol, 1968) showed changes in the region 2.5-3.5 GPa and the authors suggested that these observations might be consistent with a sluggish phase transition. IR studies of phenanthrene also indicated a phase change near 2.0 GPa (Hamann, 1978). We have recently reported (Fabbiani et al., 2004) that recrystallization of a $0.6 \mathrm{M}$ solution of phenanthrene in dichloromethane at the lower pressure of $0.7 \mathrm{GPa}$ affords a new monoclinic $\left(P 2_{1} / n\right)$ polymorph, form (III). Previously unreported structural data are presented here following new findings associated with the high-pressure studies on pyrene.

\subsection{Pyrene}

At conditions of ambient temperature and pressure, pyrene exists in two solid modifications, a stable form (I) and a metastable form (II), both crystallizing in space group $P 2_{1} / a$. The first X-ray crystal structure of form (I) was reported by Robertson \& White (1947) and was subsequently re-investigated by X-ray (Camerman \& Trotter, 1965; Allmann, 1970; Kai et al., 1978) and neutron diffraction (Hazell et al., 1972). Crystals of form (I) transform to form (II) on cooling below $110 \mathrm{~K}$ and the transition is accompanied by the shattering of the crystal. A structure of form (II) was postulated from potential energy calculations and transmission electron microscopy (TEM) by Jones et al. (1978) and this was confirmed by structure solution from high-resolution neutron powder diffraction data collected on a deuterated sample at 4.2 K (Knight et al., 1996). Single-crystal X-ray diffraction data have been obtained fairly recently (Frampton et al., 2000) by cooling a single crystal of form (I) slowly through the phase transition and by collecting data at $93 \mathrm{~K}$ on a single-crystal fragment that was preserved after shattering of the crystal. Fluorescence (Mansour \& Weinreb, 1974) and TEM (Jones \&
Cohen, 1977) measurements indicated the existence of a third phase, but no structural details have ever been published.

Volumetric measurements by Vaidya \& Kennedy (1971) pointed towards a pressure-induced phase transition in pyrene at an equilibrium transition pressure of $0.26 \mathrm{GPa}$. A transition was also observed at the slightly higher pressure of $0.4 \mathrm{GPa}$ by analysis of Raman lattice modes (Zallen et al., 1976). IR spectra (Hamann, 1978) collected in a DAC indicated a first transition at $0.3 \mathrm{GPa}$ and a second transition between 3.0 and 4.5 GPa. Spectroscopic studies at pressures below $0.4 \mathrm{GPa}$ are consistent with the I $\rightarrow$ II phase transition that has also been observed in a variable temperature and pressure neutron powder diffraction study of pyrene- $d_{10}$ (Knight $e t$ al., 1999), which showed that at ambient temperature form (I) undergoes a phase transition to the denser polymorph (II) at $c a 0.4 \mathrm{GPa}$. The phase transition had been predicted to occur at $0.19 \mathrm{GPa}$ and $300 \mathrm{~K}$ by Botoshansky et al. (2003) from a tentative pressure-temperature diagram obtained by a combination of thermodynamic and crystallographic measurements at ambient pressure.

The known polymorphism of pyrene and its high solubility in dichloromethane were deciding factors for choosing this compound for high-pressure recrystallization studies.

\section{Experimental}

\subsection{General procedures}

The Merrill-Bassett DACs used in our laboratories have a half-opening angle of $40^{\circ}$ (Merrill \& Bassett, 1974). A small piece of ruby was loaded in the DAC along each sample as a pressure calibrant. The pressure within the gasket hole was determined by measuring the ruby fluorescence on excitation with a $441.4 \mathrm{~nm}$ line from a He-Cd laser (Piermarini et al., 1975). The ruby fluorescence was dispersed and detected by a Jobin-Yvon LabRam 300 spectrometer, with a measurement precision of $\pm 0.05 \mathrm{GPa}$.

\subsection{High-pressure crystallization procedure ${ }^{1}$}

3.2.1. Naphthalene. Recrystallization experiments: A ca $2.2 \mathrm{M}$ solution of naphthalene (BDH, used as received) in dichloromethane (Fisher) was loaded at $293 \mathrm{~K}$ into a DAC equipped with $800 \mu \mathrm{m}$ culet diamonds and a tungsten gasket with a $300 \mu \mathrm{m}$ gasket hole. A single crystal was grown by cycling the temperature of the polycrystalline material obtained by sealing and pressurizing the DAC to $c a 0.4 \mathrm{GPa}$ (Fig. 2). ${ }^{1}$ This crystal was identified as the ambient-pressure phase by single-crystal diffractometry. A second single crystal grown at $c a 0.6 \mathrm{GPa}$ was also shown to correspond to the ambient-pressure phase.

Compression studies: Dissolution of naphthalene from dichloromethane at pressures higher than $0.6 \mathrm{GPa}$ could not be induced by the current heating method and so further

\footnotetext{
${ }^{1}$ Supplementary data, including a detailed experimental procedure and complete CIF for all structures determined, for this paper are available from the IUCr electronic archives (Reference: BS5035). Services for accessing these data are described at the back of the journal.
} 
studies of naphthalene were performed by compression of the single crystal grown at $0.6 \mathrm{GPa}$ from dichloromethane. Singlecrystal diffraction data were collected at 1.0 and $2.1 \mathrm{GPa}$. Broad ruby fluorescence lines indicated that above $1.0 \mathrm{GPa}$ the quality of the crystal was deteriorating and that the conditions inside the DAC were non-hydrostatic (the freezing pressure of dichloromethane is $1.33 \mathrm{GPa}$; Podsiadło et al., 2005). This is not necessarily an undesirable situation for inducing phase transformations for although non-hydrostatic stresses can in some cases suppress a phase transition (Angel et al., 2001), on other occasions uneven compression can also cause structural strain that can ultimately lead to a phase transition (Resel et al., 2004). No transition for naphthalene was observed up to $2.1 \mathrm{GPa}$. Above this pressure the 'superpressed' dichloromethane solvent froze and no single-crystal data suitable for analysis could be collected. Since the principal scope of this work was to induce the formation of a new modification of naphthalene by crystallization from solution at high pressure, no other pressure transmitting media that would allow carrying out a compressibility study to pressures higher than $2.1 \mathrm{GPa}$, and in which naphthalene is not appreciably soluble, were investigated in this instance.

3.2.2. Pyrene. Recrystallization at 0.3 and $0.5 \mathrm{GPa}$ : A ca $0.5 \mathrm{M}$ solution of pyrene (BDH, used as received) in dichloromethane (Fisher) was loaded at $293 \mathrm{~K}$ into a DAC, as described for naphthalene. Two single crystals were grown at ca 0.3 and $0.5 \mathrm{GPa}$, respectively, in the manner described above. Several temperature-annealing cycles were necessary to grow a single crystal of reasonably large size and prevent the growth of a large number of smaller single crystals nucleating from the edge of the gasket. Notwithstanding this, two smaller single crystals were also allowed to grow in the pressure cell.

Indexing of the reflections obtained from single-crystal Xray diffraction experiments gave similar unit cells with dimensions substantially different from either of the two known polymorphs of pyrene and a new polymorph, here denoted as form (III), was identified in both experiments.

\subsection{Data collection and processing}

In all experiments presented here, the DAC was mounted and centred on a Bruker SMART APEX diffractometer according to an established procedure (Dawson et al., 2004).

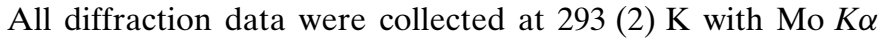

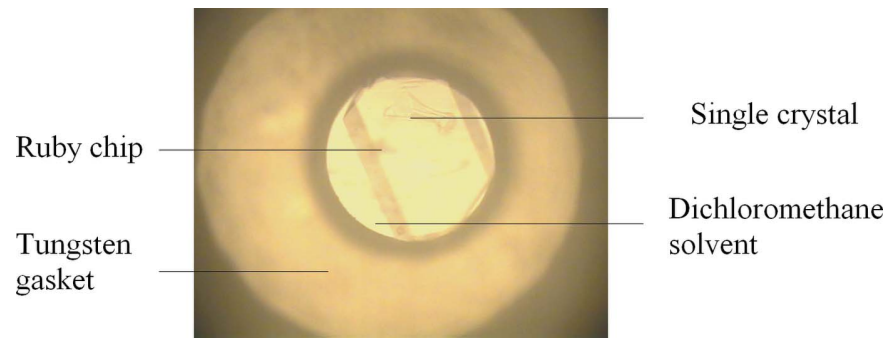

Figure 2

Optical image of a single crystal of naphthalene in a DAC at $0.4 \mathrm{GPa}$. radiation, $\lambda=0.71073 \AA$, in a sequence of eight scans (Dawson et al., 2004).

The single crystal of pyrene grown at $0.3 \mathrm{GPa}$ proved to be weakly diffracting and refinement of the unit-cell parameters in the integration step was based on only 80 reflections. This is a low number of reflections for obtaining accurate unit-cell parameters from a diffractometer equipped with an area detector. A more accurate unit cell was obtained by determining the setting angles of ten strong reflections on an EnrafNonius CAD-4 diffractometer equipped with Mo $K \alpha$ radiation $(\lambda=0.71073 \AA)$ and a point detector. The setting angles of each reflection were determined at four equivalent positions on the diffractometer to correct for any angular offsets due to sample miscentring (King \& Finger, 1979). A least-squares fit gave monoclinic unit-cell parameters in good agreement with those found with the Bruker SMART Apex CCD diffractometer, confirming that the correct unit cell had been identified, and these parameters were used during structure refinement. For the structure at $0.3 \mathrm{GPa}$, data indexing confirmed the presence of two further single-crystal domains. For the crystal grown at $0.5 \mathrm{GPa}$, data indexing identified the presence of one principal single-crystal domain and a further, smaller one that had not been identified by optical microscopy. In both cases, the twin laws relating these domains indicated essentially no reflections overlap with the principal domain. Reflections from these additional domains were too weak to be integrated. However, a reasonably good completeness ( $\mathrm{ca}$ $60 \%$ to $\theta_{\max }=20.8^{\circ}$ ) for the data collected on this lowsymmetry monoclinic crystal at $0.3 \mathrm{GPa}$ was nevertheless achieved. A considerably lower completeness of $34.4 \%$ to the same resolution was obtained for the data set collected at $0.5 \mathrm{GPa}$ due to an unfortunate orientation of the crystal.

All single-crystal data were processed according to the procedure described by Dawson et al. (2004). The sample reflections were found by using the thresholding algorithm in the SMART code or harvested manually. The orientation matrix and unit-cell geometry were determined using the program CELL_NOW (Sheldrick, 2002). Data integration and global-cell refinement were performed using the program SAINT (Bruker AXS Inc., 2003). Absorption corrections were then applied in a two-stage procedure with the programs SHADE (Parsons, 2004) and SADABS (Sheldrick, 2004). Data were subsequently merged using the program SORTAV (Blessing, 1995), as incorporated in the WinGX suite (Farrugia, 1999).

\subsection{Structure solution and refinement}

Full-matrix least-squares rigid-group refinement for all the structures was performed using the program $X L$ as incorporated in the SHELXTL suite (Sheldrick, 1997). Starting fractional coordinates from the structure determined at $92 \mathrm{~K}$ by Brock \& Dunitz (1982; CSD reference code NAPHTA06) were employed for naphthalene. The structure of the new polymorph of pyrene, which has been denoted as form (III), was solved by direct methods (XS; Sheldrick, 1997) with a pyrene molecule sitting on an inversion centre, giving half a 
Table 1

Experimental details.

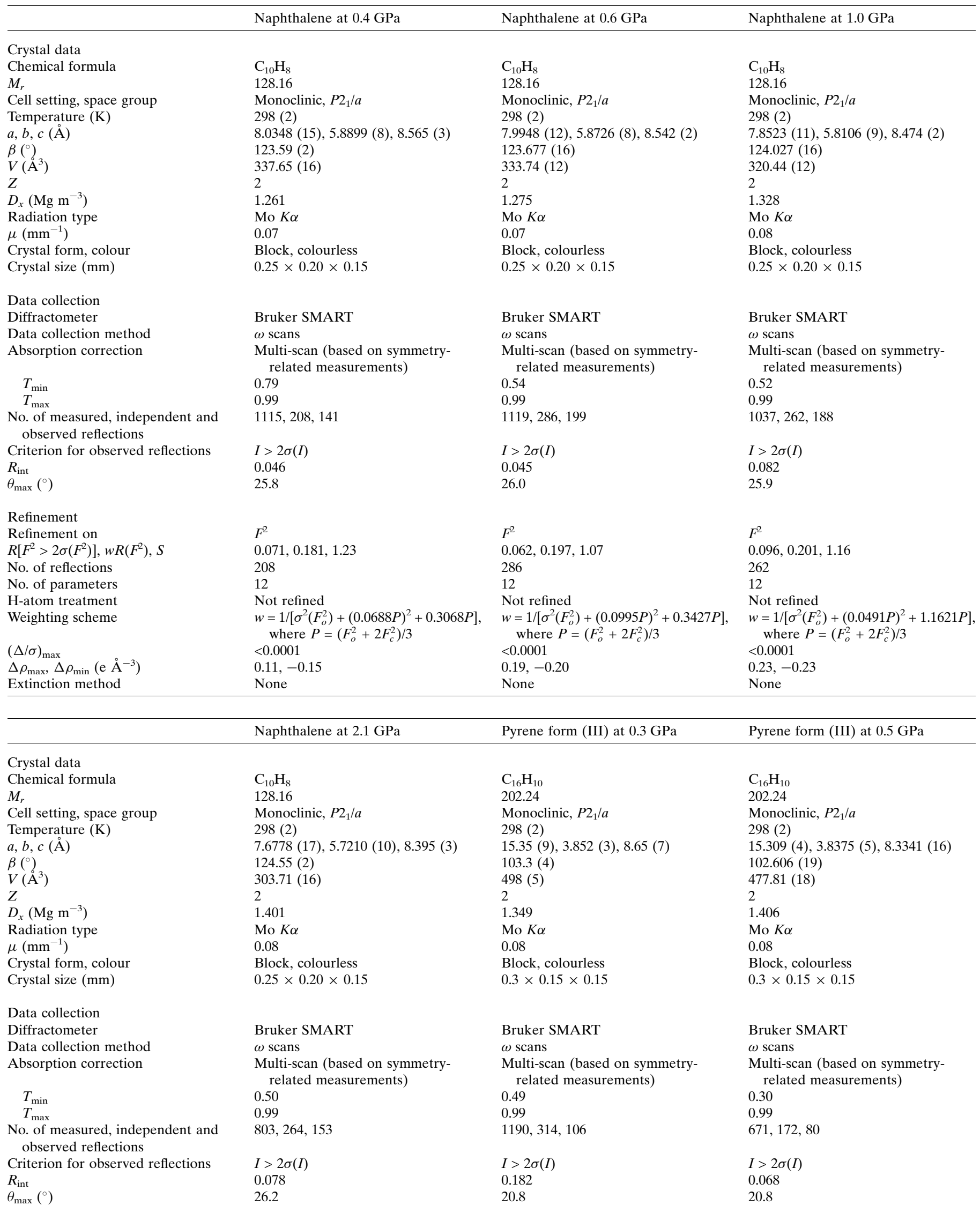


Table 1 (continued)

\begin{tabular}{|c|c|c|c|}
\hline & Naphthalene at $2.1 \mathrm{GPa}$ & Pyrene form (III) at $0.3 \mathrm{GPa}$ & Pyrene form (III) at $0.5 \mathrm{GPa}$ \\
\hline \multicolumn{4}{|l|}{ Refinement } \\
\hline Refinement on & $F^{2}$ & $F^{2}$ & $F^{2}$ \\
\hline$R\left[F^{2}>2 \sigma\left(F^{2}\right)\right], w R\left(F^{2}\right), S$ & $0.120,0.326,1.14$ & $0.118,0.408,1.08$ & $0.134,0.401,1.11$ \\
\hline No. of reflections & 264 & 314 & 172 \\
\hline No. of parameters & 12 & 9 & 8 \\
\hline H-atom treatment & Not refined & Not refined & Not refined \\
\hline Weighting scheme & $\begin{array}{l}w=1 /\left[\sigma^{2}\left(F_{o}^{2}\right)+(0.2 P)^{2}\right], \text { where } P= \\
\quad\left(F_{o}^{2}+2 F_{c}^{2}\right) / 3\end{array}$ & $\begin{array}{l}w=1 /\left[\sigma^{2}\left(F_{o}^{2}\right)+(0.2 P)^{2}\right], \text { where } P= \\
\quad\left(F_{o}^{2}+2 F_{c}^{2}\right) / 3\end{array}$ & $\begin{array}{l}w=1 /\left[\sigma^{2}\left(F_{o}^{2}\right)+(0.1514 P)^{2}+6.3887 P\right] \\
\quad \text { where } P=\left(F_{o}^{2}+2 F_{c}^{2}\right) / 3\end{array}$ \\
\hline$(\Delta / \sigma)_{\max }$ & $<0.0001$ & $<0.0001$ & $<0.0001$ \\
\hline$\Delta \rho_{\max }, \Delta \rho_{\min }\left(\mathrm{e} \AA^{-3}\right)$ & $0.34,-0.36$ & $0.21,-0.20$ & $0.28,-0.32$ \\
\hline Extinction method & None & $S H E L X L$ & None \\
\hline Extinction coefficient & - & $0.5(2)$ & - \\
\hline
\end{tabular}

Computer programs used: SMART, SAINT (Bruker AXS Inc., 2003), SHELXS97 (Sheldrick, 1997), SHELXL97 (Sheldrick, 1997), XCIF (Sheldrick, 1997), PLATON (Spek, 2003).

crystallographically independent molecule in the asymmetric unit that was refined in the non-standard $P 2_{1} / a$ setting in order to facilitate structure comparison with the known polymorphs. Owing to the weakness of high-angle data, a $\theta$ cut-off of $20.8^{\circ}$ was applied during data merging. All non-H atoms were refined isotropically; one common isotropic displacement parameter was refined for pyrene. $\mathrm{H}$ atoms were placed in calculated positions and allowed to ride on their parent atom. Distance restraints were applied for the 1,2 distances (for naphthalene and pyrene) and for three of the 1,3 distances (for pyrene) generated by symmetry about the inversion centre.

The lower quality of the naphthalene data collected at 1.0 and $2.1 \mathrm{GPa}$ was reflected in the broader reflection profiles observed and higher $R$ factors obtained. Whilst not ideal, $R$ factors of 0.119 and 0.134 for pyrene-III at 0.3 and $0.5 \mathrm{GPa}$, respectively, are within the standards for refinement of highpressure data and are sufficient to identify the main changes in crystal packing of the new high-pressure polymorph. Full refinement details are shown in Table 1.

\subsection{Decompression studies}

The question as to whether high-pressure phases are sufficiently metastable that they can be recovered at ambient conditions is of great interest, particularly if such recovered phases have industrial or technological applications. On progressive decompression from $0.5 \mathrm{GPa}$ at $293 \mathrm{~K}$, optical observation showed that the crystal of form (III) of pyrene gradually dissolved. This was in marked contrast to the disintegration of the high-pressure form of phenanthrene (Fabbiani et al., 2004). This might suggest that pyrene-(III) does not undergo a reconstructive phase transition, although at this stage it is not possible to rule out a non-reconstructive phase transition taking place prior to dissolution. Cooling of the cell to low temperatures may delay the onset of the decompression-induced dissolution and may ultimately lead to the recovery of the high-pressure phase at ambient pressure, and this is an area that is currently being explored.

\subsection{Software and other general procedures}

The structures were analysed and visualized using the programs MERCURY (Bruno et al., 2002), PLATON (Spek, 2003), as incorporated in the WIN-GX suite (Farrugia, 1999), $X P$ as incorporated in the SHELXTL suite (Sheldrick, 1997), DIAMOND (Crystal Impact, 2004), TOPOS4.0 Professional and CRYSTAL EXPLORER (Wolff et al., 2005).

\section{Results and discussion}

\subsection{Naphthalene}

No new polymorph of naphthalene was obtained by highpressure recrystallization from a dichloromethane solution and the results of a compression study are reported here.

According to the classification of Desiraju \& Gavezzotti (1989a), the crystal packing of naphthalene follows a herringbone motif, which is dominated by $\mathrm{C}-\mathrm{H} \cdots \pi$ interactions (Fig. 3a). This motif is preserved during compression to 2.1 GPa. The directional character of intermolecular interactions found in organic molecules and their generally appreciable asphericity induce significant anisotropy in the response of these crystals to pressure.

Overall, the values for lattice parameters obtained in this study are in good agreement with those of Alt \& Kalus (1982). ${ }^{2}$ The overall $18.2 \%$ increase in density over the pressure range $0.0-2.1 \mathrm{GPa}$ of naphthalene is comparable to the increase in density of anthracene, a tricyclic aromatic hydrocarbon, which shows a $c a 20 \%$ density increase over the 0.0 2.45 GPa pressure range (Oehzelt et al., 2002).

Compression of the crystallographic axes from ambient pressure to $2.1 \mathrm{GPa}$ are $6.8,4.7$ and $3.0 \%$ for $a, b$ and $c$, respectively. The value for the monoclinic $\beta$ angle increases by $1.6^{\circ}$ over the same pressure range. Rationalization of changes at a structural level as a function of pressure should be related to the linear strain in the directions of the principal axes of the strain ellipsoid rather than to the compressibilities of lattice parameters. This is because the unit cell is monoclinic and so

\footnotetext{
2 The plots that show variation of unit-cell volume, density and cell parameters with pressure have been submitted as supplementary data for this paper.
} 


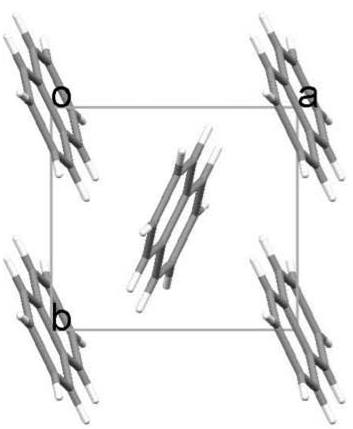

(a)

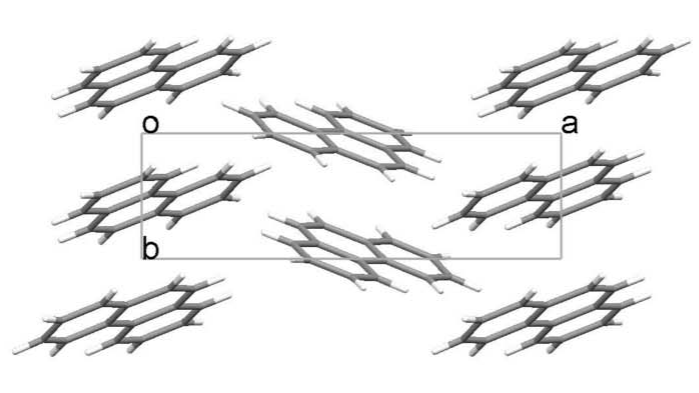

(b)

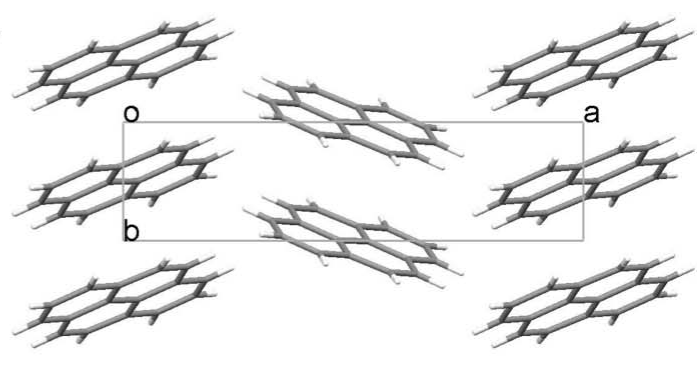

(c)

Figure 3

Crystal-packing motives of (a) naphthalene, $(b)$ phenanthrene-(III) and (c) pyrene-(III) viewed along the $c$ axis.

two of the strain tensor axes do not necessarily lie along the unit-cell axes. Calculations of linear strain in the directions of the principal axes of strain ellipsoids versus pressure (Fig. 4a) followed the procedure described in Hazen \& Finger (1982). The directions of the minimum and medium compression with respect to the cell axes are shown in Figs. 4(b) and $(c)$, respectively. The direction of medium compression coincides with the [010] direction in direct space (i.e. the $b$ axis), whereas the directions of minimum and maximum compression lie approximately along the [001] (i.e. the $c$ axis) and the [201] directions, respectively.

4.1.1. Topological analysis. The topological approach described by Peresypkina \& Blatov $(2000 a, b)$ provides a valuable tool for structure comparison. The effects of pressure on the structure of naphthalene were monitored by topological analysis using the program TOPOS4.0 Professional (Blatov et al., 2000).

When reduced to an array of molecular centroids, at ambient temperature and pressure naphthalene (NAPHTA11) is surrounded by six closest neighbours forming a distorted hexagonal planar array around the central molecule, and eight further neighbours. Spatial arrangement of these centroids and packing arrangement of the corresponding molecules are shown in Figs. 5( $a)$ and $(b)$, respectively.

Molecular centroids can be used to construct lattice Voronoi-Dirichlet polyhedra (VDPs), indicative of the global topology of packing. The coordination sequence of the corresponding lattice VDP gives the number of neighbours in the first, second and third coordination spheres, and for naphthalene this is 14-50-110, which is based on body-centred cubic (b.c.c.) topology. Taking into account only 'strong' contacts, defined by Blatov as contacts that subtend lattice VDP faces with a value for the solid angle $>1.0 \%$ (Peresypkina \& Blatov, 2000b), the coordination sequence for the lattice VDP is reduced to $12-42-92$, which is based on cubic close packed (c.c.p.) topology and depicted in Fig. 5(c). Application of pressure has the effect of 'regularizing' some VDP faces, making them more equal in size. In contrast to a smaller, more spherical-like molecule such as glycine (Dawson et al., 2005), symmetrization of the lattice VDP of naphthalene is not likely to occur even at higher pressures mainly because of the flat rod-like shape of this rigid molecule.
4.1.2. Correlating structural changes with topological analysis and strain tensors. Trends in compression of strain tensors can now be correlated with changes at the structural level. The distances between the four closest neighbours (distances 1 to 4 in Fig. $5 a$ ) that are involved in the formation of the herringbone motif have a strong component along the direction of the largest principal axis of the strain ellipsoid and consequently the motif is made 'tighter', as illustrated in Fig. 6. The second effect of pressure is to 'flatten out' the herringbone motif: the dihedral angle between pairs of molecules decreases from 52.5 (2) to $48.2(3)^{\circ}$ over the same pressure range.

Distances involving centroids 9 and 10 correspond to the distances between the parallel herringbone motifs related by translational symmetry along the $c$ axis, which is approximately parallel to the direction of minimum strain. The main effect of this compression is to bring $\mathrm{H} \cdot \mathrm{H}$ contacts closer together.

It is not surprising that the overall response of the various intermolecular interactions to compression of other organic compounds is to work cooperatively to maximize packing efficiency, increase density and minimize voids in the structure (Fig. 6), as noted by other authors (Boldyreva, 2004b; Moggach, Allan, Morrison, Parsons \& Sawyer, 2005; Moggach, Allan, Parsons, Sawyer \& Warren, 2005; Moggach, Allan, Clark, Gutmann, Parsons, Pulham \& Sawyer, 2006; Moggach, Allan, Parsons \& Sawyer, 2006; Podsiadło et al., 2005).

\subsection{Phenanthrene}

According to the classification of Desiraju \& Gavezzotti (1989a), the crystal packing of phenanthrene-(II) follows a herringbone motif, which is dominated by $\mathrm{C}-\mathrm{H} \cdots \pi$ interactions. Initial structural analysis of the ambient- and highpressure polymorph of phenanthrene and comparison with the ambient-pressure polymorph were presented elsewhere (Fabbiani et al., 2004). Inspection of the crystal packing of form (III), depicted in Fig. 3(b), shows layers held together by $\pi \cdots \pi$ interactions with a small contribution from $\mathrm{C}-\mathrm{H} \cdots \pi$ interactions.

In comparison with form (II) at ambient pressure, form (III) at $0.7 \mathrm{GPa}$ is $12 \%$ denser, indicating a more efficient packing 
of the molecules. Two effects are seen in the high-pressure phase: first, the distance between parallel planes of molecules ( $\pi \cdots \pi$ stacking) is substantially reduced; second, molecules
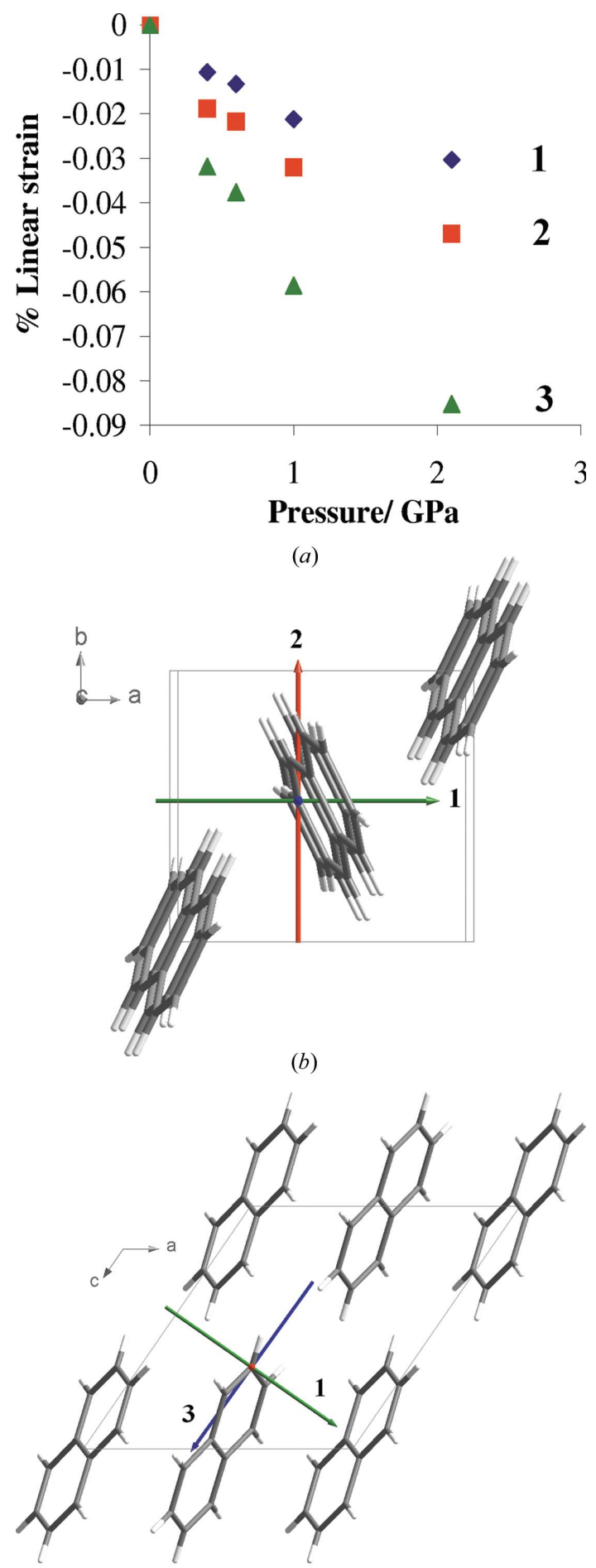

(c)

Figure 4

(a) Plot of linear strain in the directions of the principal axes of strain ellipsoids versus pressure for naphthalene. Numbering scheme: (1), maximum strain; (2), medium strain; (3), minimum strain. Orientation of the principal axes of the strain ellipsoid in naphthalene at $2.1 \mathrm{GPa}$ viewed along the direction of $(b)$ minimum $(\sim[001])$ and $(c)$ medium $([010])$ compression. The same numbering scheme was used to differentiate the different directions. between these planes overlap to a greater extent, i.e. the offset between molecules in neighbouring planes is reduced.

The coordination sequence of the lattice VDP for phenanthrene-(II) at ambient pressure is $12-42-92$, which corresponds to c.c.p. topology. For the new high-pressure phase of phenanthrene, form (III), the coordination sequence is 14-52114, which does not correspond to any of the known structure types, and the modified sequence that considers only 'strong' contacts is 10-36-79, and so is also not characteristic of any of the well known packing types. This is perhaps not surprising given the disc-like shape of the molecule.

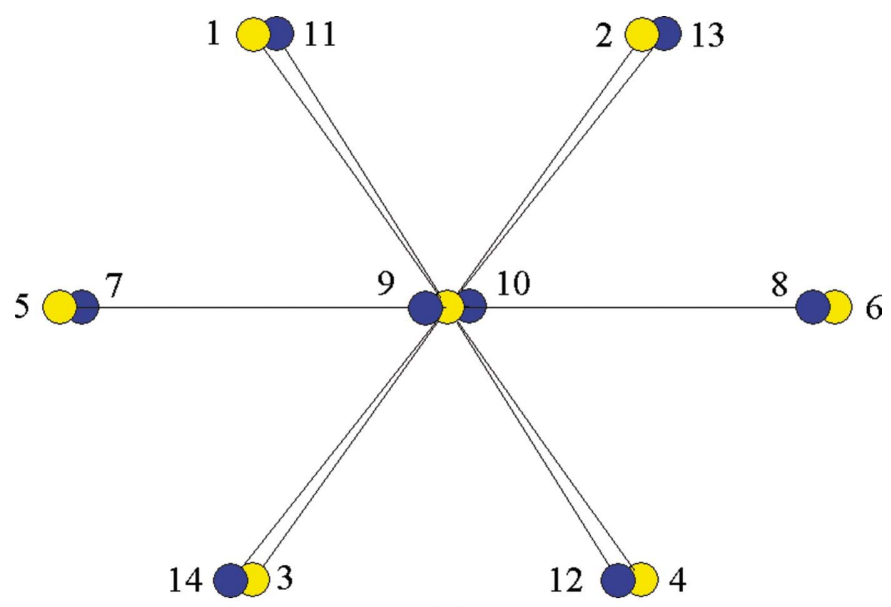

(a)
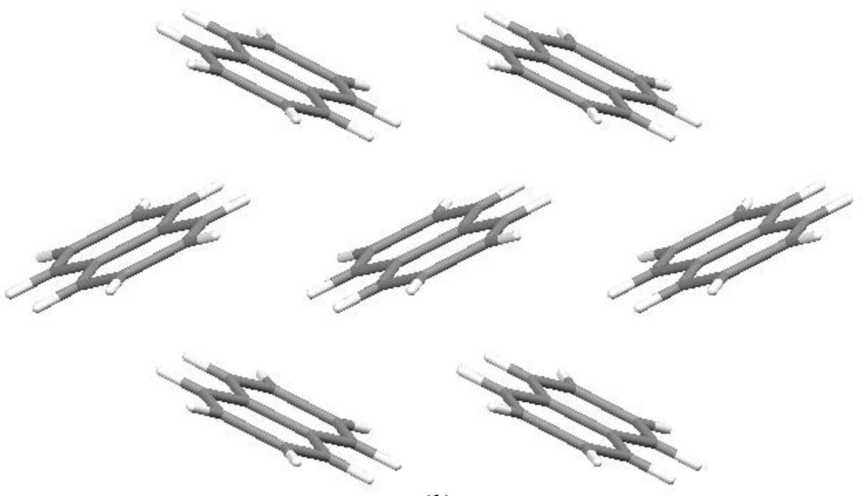

(b)
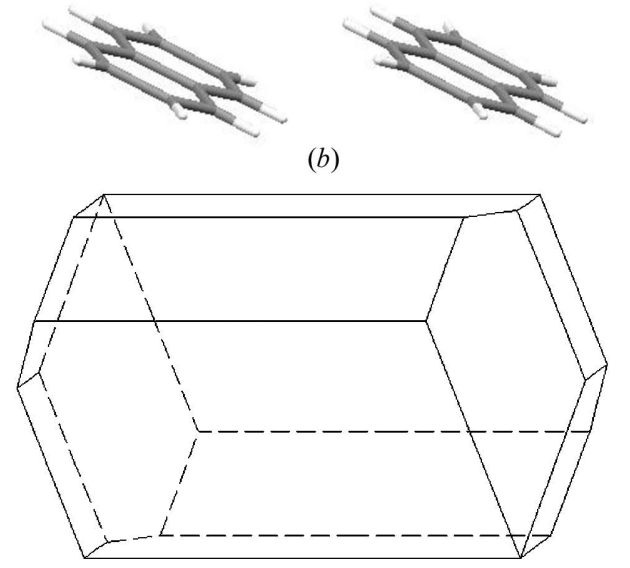

(c)

Figure 5

(a) Arrangement of the six nearest molecular centroids (numbered 1-6) around a central centroid in naphthalene, and $(b)$ their corresponding molecular packing arrangement. Further neighbours to give the full coordination sphere are numbered 7-14; (c) depicts the lattice VDP for naphthalene at $2.1 \mathrm{GPa}$. 


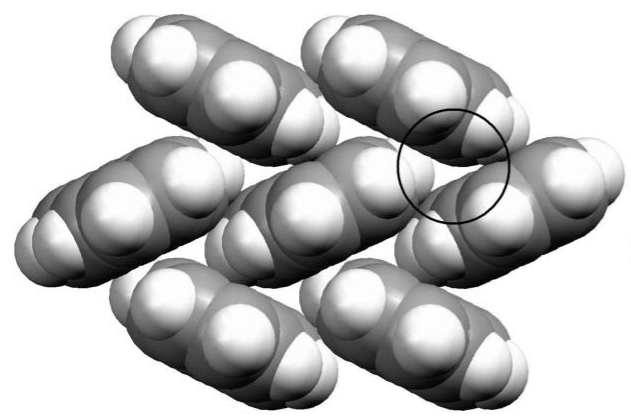

(a)

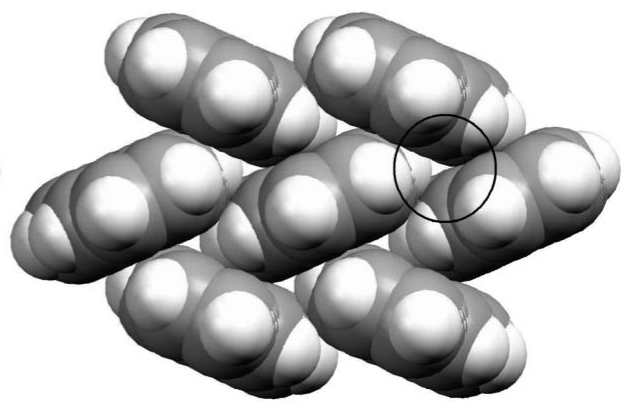

(b)

Figure 6

Space-filling plots for naphthalene at (a) ambient pressure and (b) $2.1 \mathrm{GPa}$. Examples of reduction in structural voids are shown in the circled area.

\subsection{Pyrene}

4.3.1. Forms (I) and (II). The structure of form (I) of pyrene has been reported at $113 \mathrm{~K}$ (CSD reference code PYRENE03); form (II) has been characterized at $93 \mathrm{~K}$ (CSD reference code PYRENE07). Both forms (I) and (II) belong to the sandwich-herringbone class (Desiraju \& Gavezzotti, 1989a,b): two parallel molecules (in the case of pyrene these are related by an inversion centre) are arranged in a sandwich motif via $\pi \cdots \pi$ stacking interactions, and each motif is arranged in a herringbone fashion favouring $\mathrm{C}-\mathrm{H} \cdots \pi$ interactions.

As noted by Jones et al. (1978), a small rotation of molecules around the $c$ axis of the unit cell of pyrene (I) generates a new structure that is very close in terms of cell dimensions and packing motif to form (II). This tilt is largely responsible for the noticeable change in the interplanar angle between nearest neighbours, which is $82.8^{\circ}$ for form (I) and $76.5^{\circ}$ for form (II). Interestingly, the largest changes in unitcell dimensions are associated with the $a$ and $b$ axes (see Table 2 for values), along which the sandwich-herringbone motif has strong components.

Distances between parallel molecules remain essentially unchanged at $c a 3.5 \AA$, although the offset for $\pi \cdots \pi$ stacking is slightly reduced on going from form (I) to form (II).

The six closest neighbours in forms (I) and (II) form a highly distorted hexagonal planar array around the central molecular centroid [the mean standard deviation from the best least-squares plane is $0.37 \AA$ for form (I) and $0.34 \AA$ for form (II)], which is depicted in Figs. 7(a)-(c). For forms (I) and (II), the coordination sequence is $14-50-110$, which corresponds to the coordination sequence of b.c.c. packing, although qualitatively there is very little resemblance with perfect b.c.c. topology, as expected from consideration of the disk-like shape of the molecule.

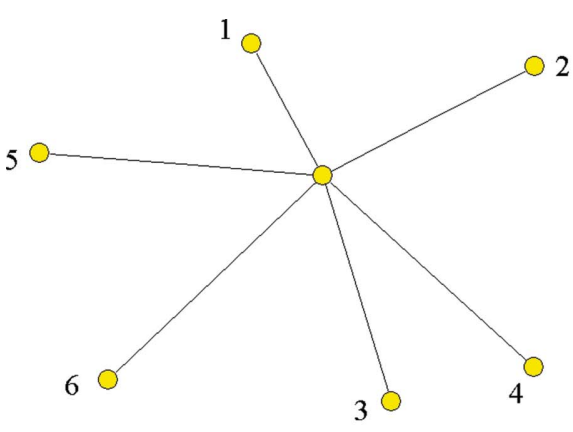

(a)

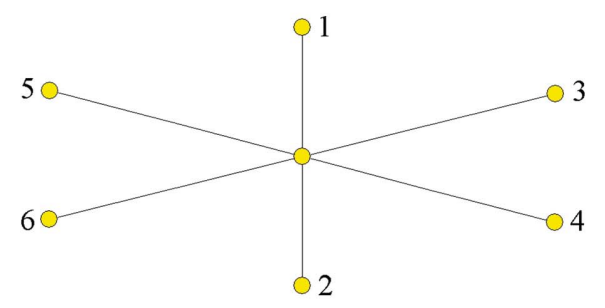

(d)

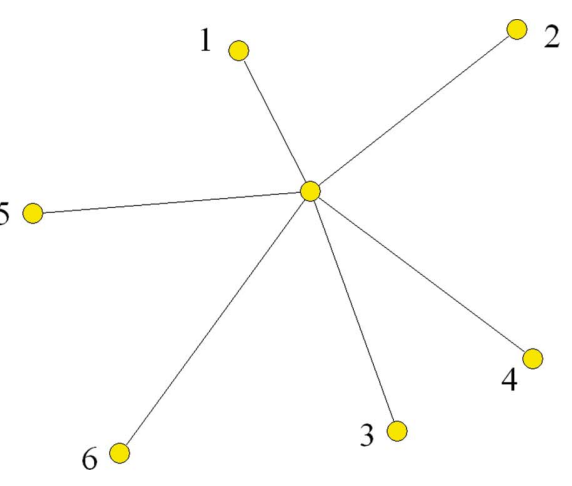

(b)

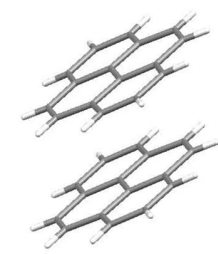

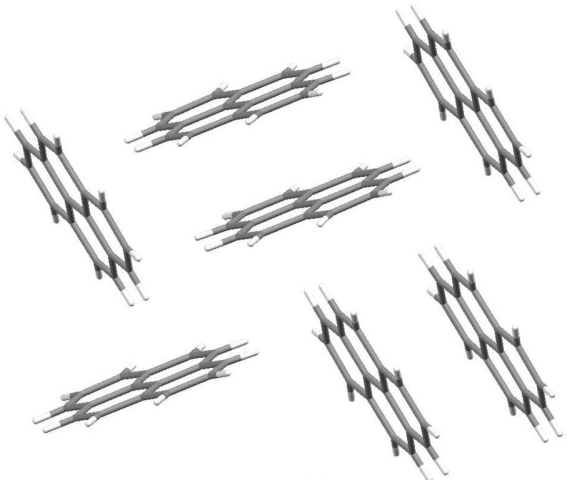

(c)

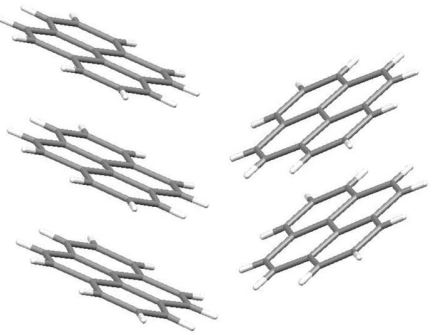

(e)

Figure 7

Arrangement of the six nearest molecular centroids around a central centroid in $(a)$ pyrene-(I), $(b)$ pyrene-(II) and (d) pyrene-(III). The corresponding molecular packing arrangements are shown in $(c)$ and $(e)$. 
Table 2

Unit-cell parameters and intermolecular interplanar angles for naphthalene and the polymorphs of phenanthrene and pyrene.

\begin{tabular}{|c|c|c|c|c|c|c|}
\hline & Naphthalene & Phenanthrene (II) & Phenanthrene (III) & Pyrene (I) & Pyrene (II) & Pyrene (III) \\
\hline CSD reference code & NAPHTA $10^{a}$ & PHENAN $13^{b}$ & PHENAN14 $^{c}$ & PYRENE03 $^{d}$ & PYRENE07 ${ }^{e}$ & $-^{f}$ \\
\hline Crystal system & Monoclinic & Monoclinic & Monoclinic & Monoclinic & Monoclinic & Monoclinic \\
\hline Space group & $P 2_{1} / a$ & $P 2_{1}$ & $P 2_{1} / n$ & $P 2_{1} / a$ & $P 2_{1} / a$ & $P 2_{1} / a$ \\
\hline$a(\AA)$ & $8.213(2)$ & $8.472(4)$ & $12.937(3)$ & $13.532(1)$ & $12.358(6)$ & $15.35(9)$ \\
\hline$b(\AA)$ & $5.9727(13)$ & $6.166(4)$ & $3.8218(5)$ & $9.159(1)$ & $10.020(4)$ & $3.852(3)$ \\
\hline$c(\AA)$ & $8.6745(18)$ & $9.467(5)$ & $17.693(6)$ & $8.387(1)$ & $8.260(4)$ & $8.65(7)$ \\
\hline$\beta\left({ }^{\circ}\right)$ & $123.388(18)$ & $98.01(5)$ & $99.13(2)$ & $100.25(1)$ & $96.48(4)$ & $103.3(4)$ \\
\hline Temperature (K) & 239 & 293 & 293 & 113 & 93 & 293 \\
\hline Interplanar angle $\left(^{\circ}\right)$ & 52 & 58 & 52 & 83 & 76 & 60 \\
\hline
\end{tabular}

References: (a) Brock \& Dunitz (1982); (b) Kay et al. (1971); (c) Fabbiani et al. (2004); (d) Kai et al. (1978); (e) Frampton et al. (2000); (f) this work.

4.3.2. Form (III) at 0.3 and $0.5 \mathrm{GPa}$. Direct inspection of the crystal packing of the new high-pressure polymorph, form (III), depicted in Fig. 3(c), shows layers held together by $\pi \cdots \pi$ interactions with a small contribution from $\mathrm{C}-\mathrm{H} \cdots \pi$ interactions. The new polymorph at $0.3 \mathrm{GPa}$ has a density that is $c a$ $6.1 \%$ higher than that of form (I) at ambient pressure and temperature (CSD reference code PYRENE01); at $0.5 \mathrm{GPa}$ the increase is found to be $c a 10.6 \%$. The substantial increase in density is comparable to the increase observed for the highpressure polymorph of phenanthrene, as noted earlier.

4.3.3. Topological analysis. As in the case of phenanthrene, the increase in the density of the high-pressure phase of pyrene is indicative of more efficient packing of the molecules. In the high-pressure phase, each molecule has six nearest neighbours that give rise to a distorted hexagonal planar array (Figs. $7 d$ and $e$ ). Overall, the topology resembles a distorted c.c.p. arrangement. This is confirmed by the fact that the coordination sequence is $12-42-92$. Centroids 1 and 2 correspond to pyrene molecules involved in $\pi \cdots \pi$ stacking related by translation along the $b$ axis. Although the distances between parallel molecules are essentially unchanged, different types of offsets to $\pi \cdots \pi$ stacking are observed for the three polymorphs, as will be illustrated in the analysis of Hirshfeld surfaces. The direction of the largest principle axis of the strain ellipsoid was found to lie approximately along the $c$ axis. This compresses by $c a 3.7 \%$ from 0.3 to $0.5 \mathrm{GPa}$ with the result of a reduction of voids in the structure.

\subsection{Classification of the crystal structures of the high- pressure polymorphs of pyrene and phenanthrene}

In their pioneering work, Desiraju \& Gavezzotti (1989a,b) divided PAHs into four structural types: $\beta, \gamma$, herringbone and sandwich-herringbone structures. According to the authors, the key parameters to distinguish between the four structure types are the shortest cell axis and the interplanar angle (Fig. 8 ), defined as the angle between the mean plane of one molecule and that of its nearest neighbours (this model shall be referred to as the 'geometrical model'); in particular, the authors stated 'the shortest axis is in fact crystal structure defining while the two other cell axes are merely a function of individual molecular geometries'. They found that the over- whelming majority of crystal structures of PAHs are monoclinic, with the shortest axis coinciding with the unique axis.

The stable polymorph of phenanthrene, at ambient pressure and ambient temperature, phase (II), is no exception, and with a $b$ axis of 6.166 (4) $\AA$ and an interplanar angle of $58^{\circ}$, it belongs to the herringbone-type of structures, dominated by $\mathrm{C}-\mathrm{H} \cdots \pi$ interactions (Desiraju \& Gavezzotti, 1989b). Values for the lattice parameters and interplanar angles for the polymorphs of phenanthrene and pyrene are given in Table 2. According to the geometrical model, the new high-pressure phase of phenanthrene, form (III), with a $b$ axis of 3.8218 (5) $\AA$, would belong to the $\beta$-type structures, characterized by layered structures made of 'graphitic' planes. On the other hand, this would make form (III) a distinct 'outlier' in this class, as shown in Fig. 8, since an interplanar angle of

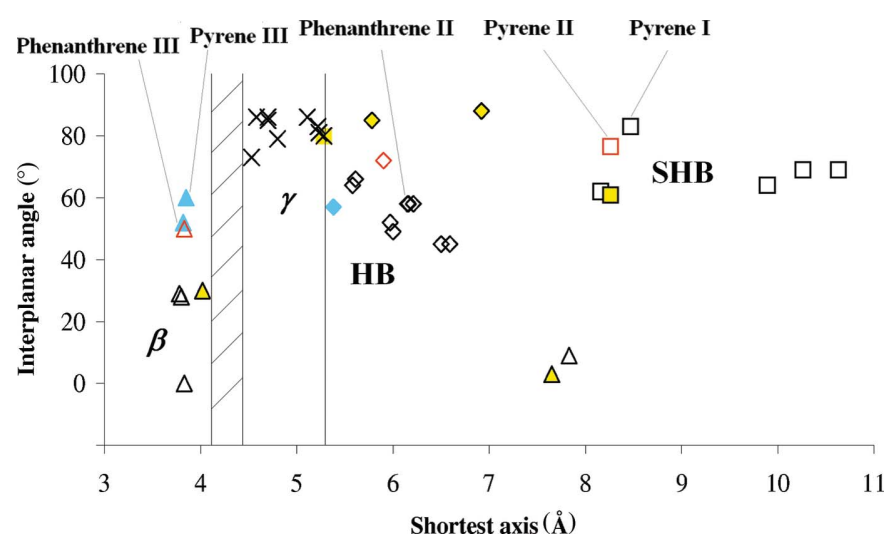

Figure 8

Intermolecular interplanar angle versus shortest cell axis in the crystal structures of 31 of the 32 PAHs analysed by Desiraju \& Gavezzotti (1989b) and their classification into four-structure types: $\beta$ (triangles), $\gamma$ (crosses), herringbone (HB, rhombi) and sandwich-herringbone (SHB, squares) structures. Orthorhombic structures are highlighted in yellow. The light blue rhomb is the high-pressure monoclinic phase of benzene. Seven structures have been added to the 31 originally classified: the highpressure forms of pyrene and phenanthrene (blue triangles), benzodicoronene (open red triangle), $\beta$-perylene (open red rhomb), pyrene-(II) (open red square) and the orthorhombic polymorph of dibenzanthracene (black square highlighted in yellow). For structural parameters of naphthalene and the polymorphs of phenanthrene and pyrene discussed in the main text, see Table 2. 
$52^{\circ}$ is atypical for this class of structures, which typically exhibit values below $30^{\circ}$, and in fact falls in the region of herringbone-type structures.

Desiraju \& Gavezzotti also stated that '...the shortest crystallographic axis is always a screw-axis direction. This axis is, therefore, a key parameter in separating packing types and defines the crystal structure' (Desiraju \& Gavezzotti, 1989a). In their analysis, the authors always selected values for the shortest unit-cell axis, but we note here that in the case of pyrene-(I) and pyrene-(II) this axis does not coincide with the unique axis - the shortest axis is in fact the $c$ axis - and so this would appear to run counter to the established trend. On closer inspection of other PAHs structures reported in their papers, dibenzanthrancene, perylene, benzperylene, dinaphthoanthracene, benzopyrene and benzobisanthrene were also found not to follow this rule. It is therefore concluded that whilst the monoclinic $b$ axis is always crystal defining, it is not necessarily always the shortest one. In the case of pyrene, two types of analysis could therefore be envisaged, based on the consideration of the length of the shortest axis or of the unique axis. The shortest $(c)$ axis and interplanar angles are 8.387 (1) $\AA$ and $82.8^{\circ}$ for form (I) (CSD reference code PYRENE03) and 8.260 (4) $\AA$ and $76.5^{\circ}$ for form (II) (CSD reference code PYRENE07). The unique $(b)$ axis is 9.159 (1) $\AA$ for form (I) and 10.020 (4) $\AA$ for form (II). According to both types of analysis both structures belong to the sandwich-herringbone class, a hybrid between herringbone and $\gamma$ structures, in which both $\mathrm{C}-\mathrm{H} \cdots \pi$ and $\pi \cdots \pi$ interactions are present.

With a $b$ axis of 3.852 (3) $\AA$ and an interplanar angle of $60^{\circ}$ (Fig. 8), the classification of pyrene-(III) is remarkably similar to that of phenanthrene-(III).

In the analysis by Desiraju \& Gavezzotti $(1989 a, b)$, the authors identified a number of other structures which do not fit their classification. One example is the high-pressure monoclinic form of benzene (Fig. 8), which has an interplanar angle typical of a herringbone structure, but a rather short unique axis more typical of the $\gamma$-type. At ambient pressure, the structures of diperinaphthyleneanthracene (CSD reference code NAPANT01) and tetrabenzoperylene (TBZPER) are classified as $\beta$-type by virtue of their small interplanar angles of 9 and $3^{\circ}$, respectively, but have values for the shortest axis of 7.803 (1) and 7.656 (2) $\AA$, respectively, which are highly atypical of $\beta$-type structures. However, tetrabenzoperylene crystallizes in the orthorhombic crystal system and the authors acknowledged some possible limitations of their geometrical model when applied to this type of system.

It therefore appears that both new high-pressure polymorphs of phenanthrene and pyrene represent deviations from the geometrical model by Desiraju \& Gavezzotti. In both cases, the interplanar angle would suggest herringbone-type structures, but based on the length of the unique (and shortest) axis, classification as $\beta$-type structures is suggested. In the preceding section, analysis of crystal packing and topology showed unequivocally that these two structures are indeed dominated by $\pi \cdots \pi$ stacking, and so should be classified as $\beta$-type structures. Hence, these results show that for the limited number of high-pressure structures (phenanthrene, pyrene and benzene) it is the length of the unique (shortest) axis that is more indicative of the structure type rather than the interplanar angle. These deviations from the model of Desiraju \& Gavezzotti is perhaps not surprising since their trends relied on the geometrical parameters and interactions obtained at ambient pressure. At high pressure, the hierarchy of intermolecular interactions can be very different, thereby leading to very different structural types. Although the sample of high-pressure structures of PAHs is only small, it does appear that high pressure may favour structures involving $\pi \cdots \pi$ interactions.

\subsection{Decoding intermolecular interactions: Hirshfeld surfaces and fingerprint plots}

A comparison of Hirshfeld surfaces and fingerprint plots produced with CRYSTAL EXPLORER (Wolff et al., 2005) is presented in this section. Particular attention is given to fingerprint plots, which provide a concise summary of the intermolecular interactions occurring in the crystal by mapping the fraction of points on the corresponding Hirshfeld surface as a function of the closest distances from the point to nuclei interior $\left(d_{i}\right)$ and exterior $\left(d_{e}\right)$ to the surface. Colours in these plots range from blue (relatively few points) through green (moderate fraction) to red (many points). Curvedness has been mapped on the Hirshfeld surfaces between -4.0 (red) and +0.4 (blue), and shape index between -1.0 (red) and +1.0 (blue). A range of 1.0 (red) and 2.5 (blue) for mapping $d_{e}$ was employed here.

The uniqueness of Hirshfeld surfaces and the corresponding fingerprint plots for any crystal structure makes them a powerful tool for elucidating and comparing intermolecular interactions, particularly when comparing the same molecule in different crystal environments, as well as for spotting common features/trends in specific classes of compounds. Being unique single colour plots, they provide a major practical advance in the description of crystal structures and complement other tools currently available for the systematic description and analysis of organic molecular crystal structures, e.g. graph-set analysis (Etter et al., 1990; Bernstein et al., 1995) and topological analysis. Fingerprint plots have been shown (Spackman \& McKinnon, 2002; McKinnon et al., 2004) to provide a rapid visual tool for discriminating between the four PAHs structure types and to identify examples where molecules exhibit features characteristic of more than one structural type.

4.5.1. Naphthalene. Hirshfeld surfaces for naphthalene at conditions of ambient pressure and $239 \mathrm{~K}$ (CSD reference code NAPHTA10) have been recently described by McKinnon et al. (2004) and are not shown here. The authors showed how $\mathrm{C}-\mathrm{H} \cdots \pi$ interactions manifest themselves most visibly as a broad red depression on the $d_{e}$ surface. These interactions give rise to characteristic 'wings' on the corresponding fingerprint plot around $d_{e}=1.2 \AA-d_{i}=1.8 \AA$ and $d_{e}=1.8 \AA-d_{i}=1.2 \AA$. These features are visible in the Hirshfeld surfaces and fingerprint plot for naphthalene at 
2.1 GPa and $293 \mathrm{~K}$ depicted in Figs. 9 and 10(e). Only one side of the surfaces is shown as the molecule resides on an inversion centre.

Comparison of the ambient-pressure (at ambient temperature, CSD reference code NAPHTA11) and high-pressure structures is made on the basis of fingerprint plots since these summarize the differences most visibly. A series of fingerprint plots ranging from ambient pressure to $2.1 \mathrm{GPa}$ is shown in Fig. 10. As expected, the plots maintain their overall shape and characteristic features, since no drastic structural change, e.g. a change associated with a phase transition, is encountered over this pressure range. The finding that increasing density is observed with increasing pressure manifests itself in the progressive shift of the plots to lower $d_{e}$ and $d_{i}$ values. This

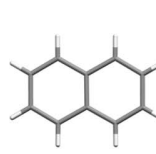

Figure 9

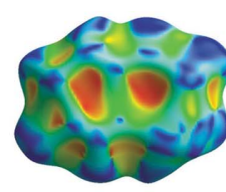

(b)

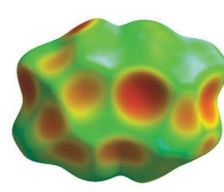

(c)
Hirshfeld surfaces for naphthalene at $2.1 \mathrm{GPa}$ and $293 \mathrm{~K}$. The molecule is shown with the Hirshfeld surface mapped with $(a)$ curvedness, $(b)$ shape index and $(c) d_{e}$. shift can be deconvoluted for various interactions in turn: for example, $\mathrm{C}-\mathrm{H} \cdots \pi$ interactions become visibly shorter, as indicated by the position of the characteristic 'wings' mentioned earlier, and this mirrors the results of the topological analysis presented earlier, where the direction of maximum compression was associated with these interactions. $\mathrm{H} \cdots \mathrm{H}$ contacts (corresponding to the pointed feature at the bottom left of the plots) also become considerably shorter, from just below $1.2 \AA$ at ambient pressure to just above $1.0 \AA$ at $2.1 \mathrm{GPa}$. The upper part of the plots, where data points are scarce and $d_{e}$ is larger than $d_{i}$, is representative of voids in the structure: these voids also follow the general trend of contraction, as it was shown in Fig. 6.

4.5.2. Phenanthrene-(II). Hirshfeld surfaces for phenanthrene at conditions of ambient temperature and pressure (CSD reference code PHENAN13) have recently been presented by McKinnon et al. (2004) and are not depicted here. The corresponding fingerprint plot is illustrated in Fig. $11(d)$. The herringbone structure of phenanthrene-(II) is also dominated by $\mathrm{C}-\mathrm{H} \cdots \pi$ contacts and these manifest themselves as described for naphthalene.

4.5.3. Phenanthrene-(III). Hirshfeld surfaces and the fingerprint plot for phenanthrene-(III) at $0.7 \mathrm{GPa}$ are shown

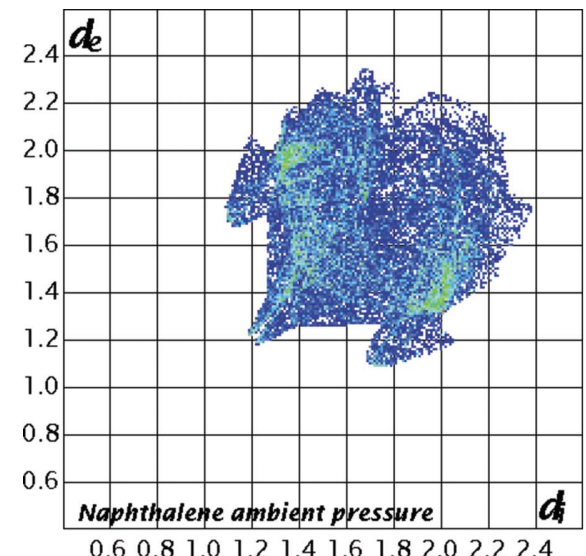

(a)

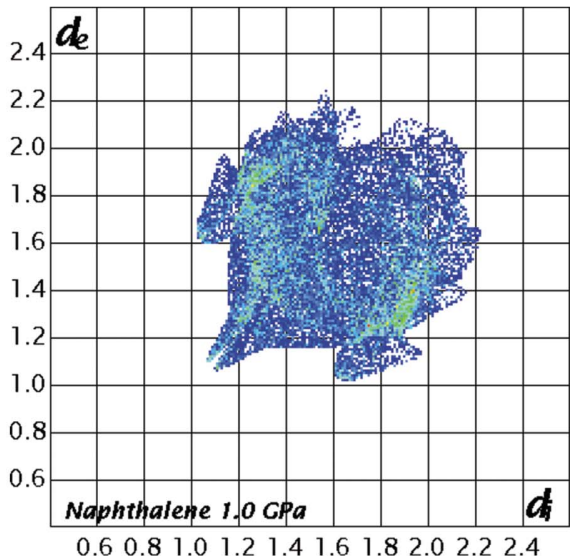

(d)

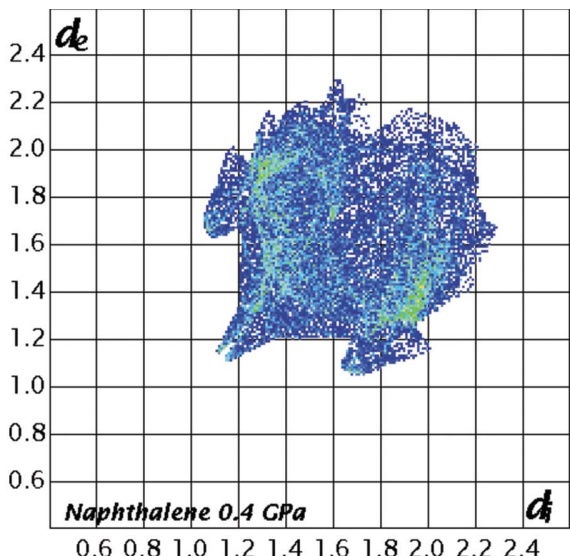

(b)

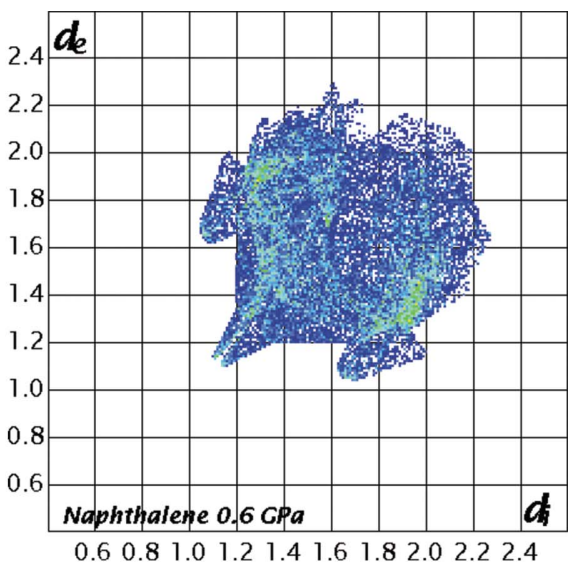

(c)

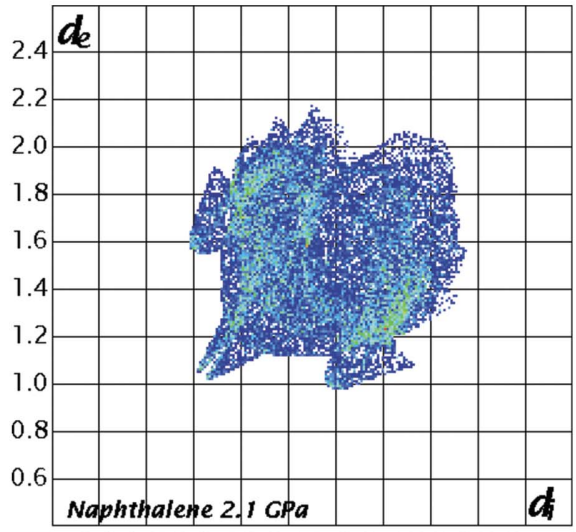

$\begin{array}{llllllllllll}0.6 & 0.8 & 1.0 & 1.2 & 1.4 & 1.6 & 1.8 & 2.0 & 2.2 & 2.4\end{array}$

(e)

Figure 10

Fingerprint plots for naphthalene at (a) ambient pressure (CSD reference code NAPHTA11), (b) 0.4 GPa, (c) 0.6 GPa, (d) $1.0 \mathrm{GPa}$ and (e) $2.1 \mathrm{GPa}$. 
in Fig. 11. The mode of packing of form (III) was earlier contrasted to the herringbone-type of packing of form (II). On the basis of the length of the unique axis, the new high-pressure form (III) should belong to the $\beta$-type structures. These different modes of packing are clearly illustrated in Fig. 11.

The fingerprint plot for form (III) is remarkably different from that of the herringbone structures of form (II). Fingerprint plots for pure $\beta$-type structures are overall noticeably different from those of the other structure types: the prominent 'wings' indicative of $\mathrm{C}-\mathrm{H} \cdots \pi$ contacts are absent and the dominant contact between molecules is $\pi \cdots \pi$ stacking, shown as a red area around $d_{e}=d_{i} \simeq 1.8 \AA$. In Fig. 11, the 'wings' indicative of $\mathrm{C}-\mathrm{H} \cdots \pi$ contacts on the fingerprint plot of form (III) are indeed absent and the broad depressions above and below the aromatic ring in the shape index surface and $d_{e}$ surfaces are moved to the edges of the surface, thereby indicating the presence of limited $\mathrm{C}-\mathrm{H} \cdots \pi$ (peripheral) interactions. The dominant contact between molecules is now $\pi \cdots \pi$ stacking, visible as a red/green area on the fingerprint plot diagonal around $d_{e}=d_{i} \simeq 1.78 \AA$ and corresponding to an interlayer distance of $c a 3.5 \AA$. $\pi$. . $\pi$ stacking is evident on the
Hirshfeld surface as a large flat region across the molecule, and is most clearly visible on the curvedness surface. The pattern of red and blue triangles on the same region of the shape index surface is another characteristic of $\pi \cdots \pi$ stacking (McKinnon et al., 2004). The significantly higher density of form (III) is reflected by the compaction of the fingerprint plot to lower $d_{e}$ and $d_{i}$ values in comparison with the plot of form (II) at ambient pressure.

4.5.4. Pyrene-(I) and pyrene-(II). Fingerprint plots and Hirshfeld surfaces for pyrene-(I) at ambient pressure and room temperature (CSD reference code PYRENE02) have been reported and discussed by McKinnon et al. (2004). A summary of their work is reported here with reference to the ambient-pressure structure at $113 \mathrm{~K}$ (CSD reference code PYRENE03) that was discussed earlier and the analysis is extended to form (II) at $93 \mathrm{~K}$ (CSD reference code PYRENE07). Both forms (I) and (II) belong to the sandwichherringbone type of structures and this is clearly visible in the corresponding Hirshfeld surfaces and fingerprint plots of Fig. 12. One side of the molecule is involved in $\pi \cdots \pi$ stacking and
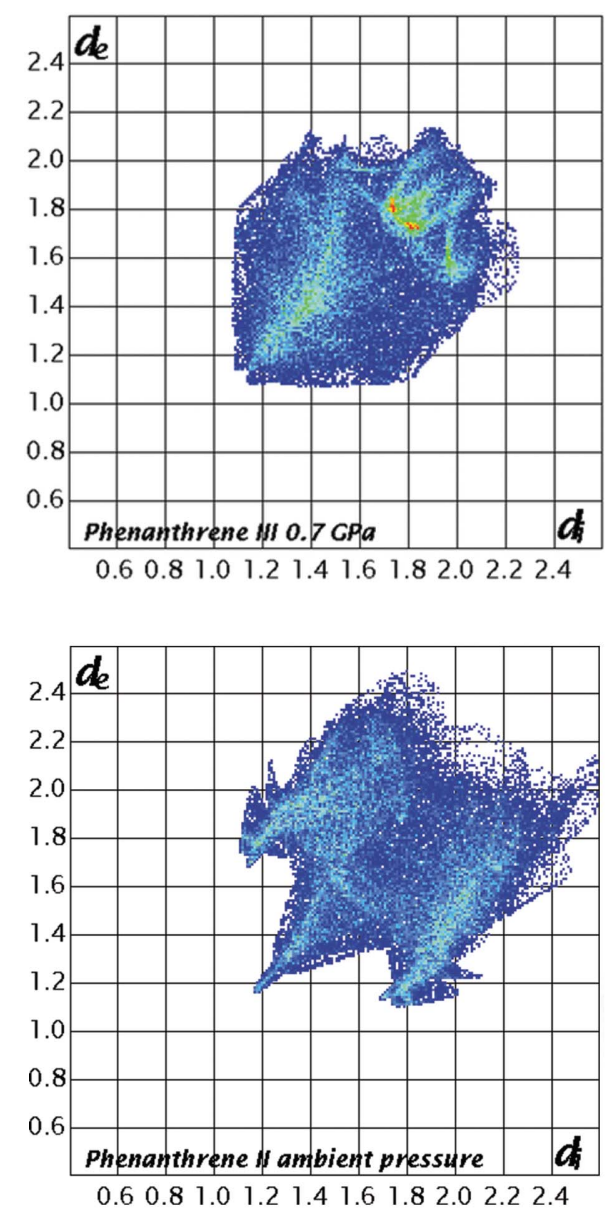

$(d)$
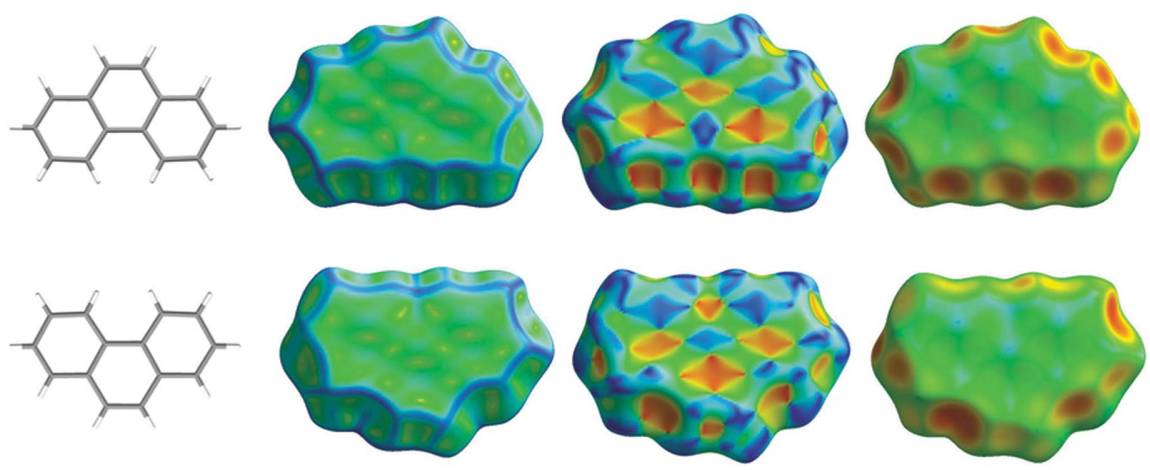

(a)

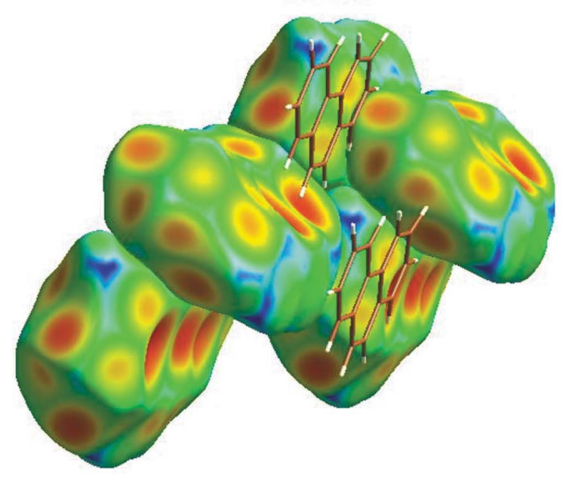

(b)

(c)

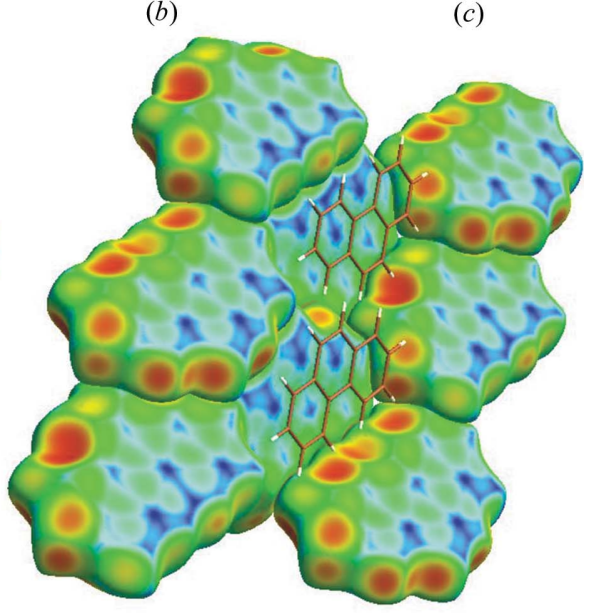

(f)

Figure 11

Fingerprint plot and Hirshfeld surfaces for phenanthrene-(III) at $0.7 \mathrm{GPa}$ (CSD reference code PHENAN14). The molecule is shown with the Hirshfeld surface mapped with $(a)$ curvedness, $(b)$ shape index and $(c) d_{e}$. Fingerprint plot $(d)$ and crystal-packing diagram for $(e)$ phenanthrene-(II) at ambient pressure (CSD reference code PHENAN13) and $(f)$ phenanthrene-(III) at $0.7 \mathrm{GPa}$, with Hirshfeld surfaces mapped with $d_{e}$ illustrating the profoundly different modes of packing of the two polymorphs. 
shows a corresponding flat Hirshfeld surface, whilst the other has a prevalence of $\mathrm{C}-\mathrm{H} \cdots \pi$ interactions.

For forms (I) and (II) the pattern of alternating red and blue triangles with local threefold symmetry on the same region of the shape-index surface is indicative of offset $\pi \cdots \pi$ stacking interactions characteristic of graphite-like layers. The change in the orientation of these triangles in form (II) is indicative of the slight change in the offset discussed in the topological analysis section.

The wings characteristic of $\mathrm{C}-\mathrm{H} \cdots \pi$ contacts are well separated on the fingerprint plot of form (I), whilst they are almost overlapping for form (II) and this is mirrored by the four large depressions on the $d_{e}$ surfaces being more evenly coloured. The fact that similar distances are associated with these two contacts in form (II) is a direct consequence of the change in the tilt of the herringbone motif.

For form (I) head-to-head $\mathrm{H} \cdots \mathrm{H}$ contacts at $d_{e}=d_{i}=$ $1.02 \AA$ give rise to the pointed feature on the diagonal at the bottom left: this contact for form (II) is at a longer distance of $1.16 \AA$ and appears to be split on the fingerprint, indicating that the shortest contact is between three atoms, rather than between two.
4.5.5. Pyrene-(III). The new high-pressure polymorph of pyrene, form (III), exhibits packing features typical of $\beta$ structures with limited contributions from $\mathrm{C}-\mathrm{H} \cdots \pi$ interactions. These criteria are met in the fingerprint plots and Hirshfeld surfaces for pyrene-(III) at $0.3 \mathrm{GPa}$ shown in Fig. 13, as discussed earlier for the high-pressure polymorph of phenanthrene (only one side of the surfaces is shown as the molecule resides on an inversion centre).

The offset to perfect $\pi \cdots \pi$ stacking produces an alternating rhomboidal pattern of blue and red regions of the shape index. This type of stacking, also found in the structure of hexabenzocoronene (McKinnon et al., 2004), is indicative of a reduced offset in comparison with graphite-like stacking. The centres of the four rings of pyrene are shown as deep blue spots on the upper part of the $d_{e}$ surface.

Comparison between the fingerprint plots of forms (I), (II) and (III) of pyrene shows very clearly the highest density of form (III), with the plot being spread over lower $d_{e}$ and $d_{i}$ values. The fingerprint of pyrene-(III) at $0.5 \mathrm{GPa}$ is shown in Fig. 14(a). The increase in density from 0.3 to $0.5 \mathrm{GPa}$ is also apparent at higher $d_{e}$ and $d_{i}$ values. At $0.5 \mathrm{GPa}$, 'void' regions are noticeably reduced in number and in size.
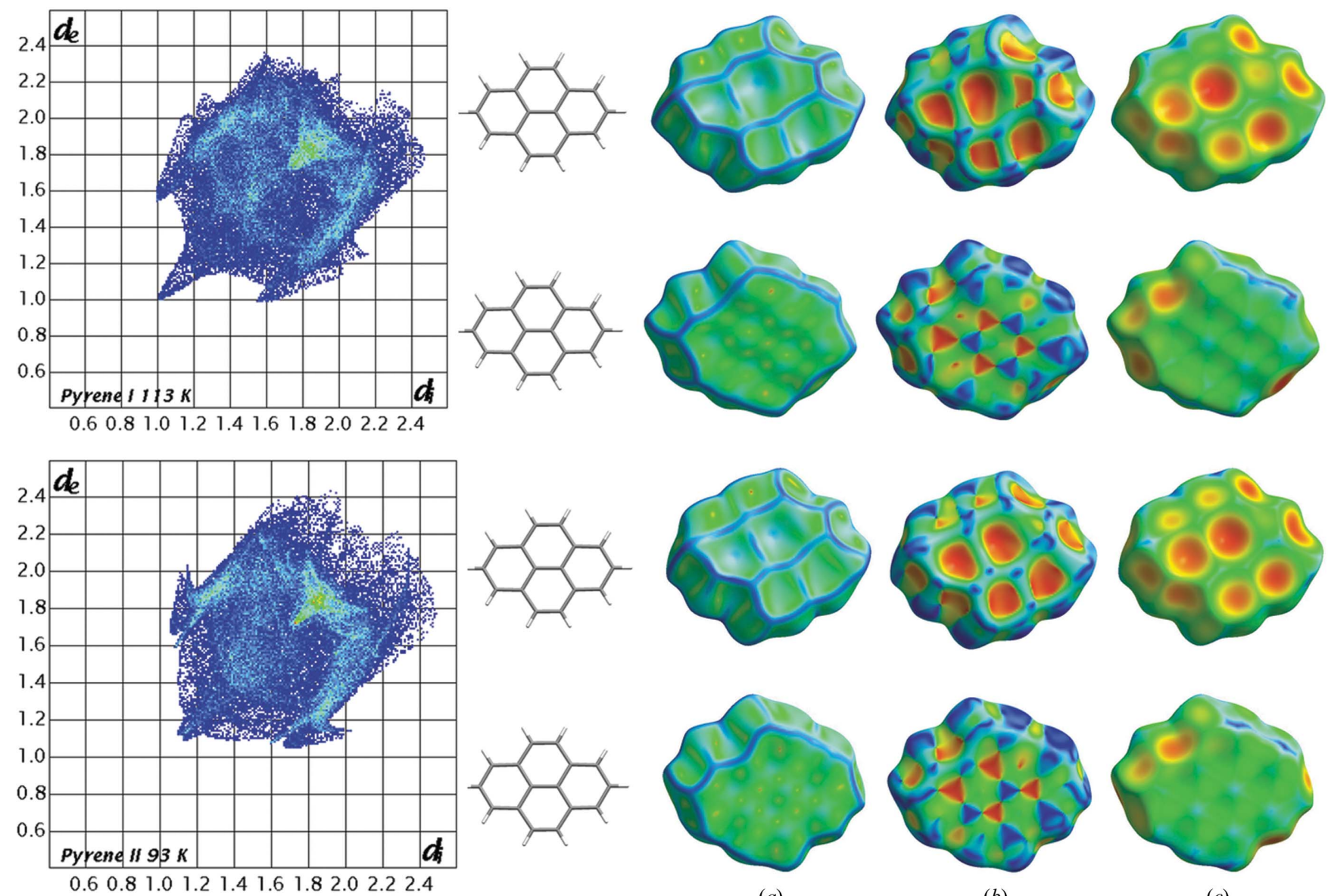

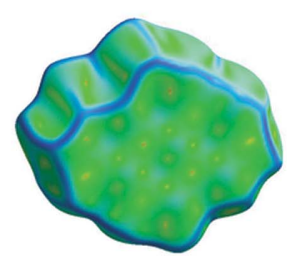

(a)

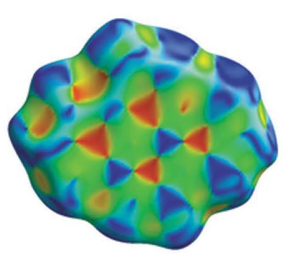

(b)

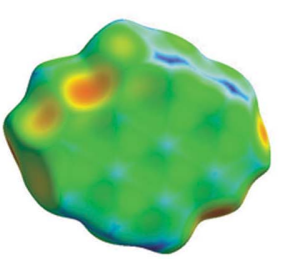

(c)

Figure 12

Hirshfeld surfaces and fingerprint plot for pyrene-(I) at $113 \mathrm{~K}$ (CSD reference code PYRENE03) and pyrene-(II) at $93 \mathrm{~K}$ (CSD reference code PYRENE07). The molecule is shown with the Hirshfeld surface mapped with (a) curvedness, $(b)$ shape index and $(c) d_{e}$. 


\subsection{Overall discussion of phenanthrene-(III) and pyrene-(III)}

Overall, the fingerprint plots of phenanthrene-(III) and pyrene-(III) show features that are typical of $\beta$-type structures (an example of which is given in Fig. $14 b$ for anthrabenzonaphthopentacene), with only some limited contributions from $\mathrm{C}-\mathrm{H} \cdots \pi$ interactions.

The fingerprint plots of phenanthrene-(III) and pyrene(III) are remarkably similar to that of benzodicoronene, depicted in Fig. 14(c). Benzodicoronene, a 15-membered ring $\mathrm{PAH}$, also represents an outlier with respect to the geometrical model by Desriraju and Gavezzotti reported in Fig. 8. In fact, similarly to phenanthrene-(III) and pyrene-(III), benzodicoronene (CSD reference code YOFCUR; Goddard et al., 1995) also exhibits a short axis [3.823 (1) $\AA$ ], whose value is typical of $\beta$-type structures, but an interplanar angle $\left(50.2^{\circ}\right)$ that is larger than normally found for this structural class.

The outlier status of benzodicoronene was also pointed out by McKinnon et al. (2004), who classified it as a $\gamma$-type structure, but noted that the fingerprint showed almost no contribution from $\mathrm{C}-\mathrm{H} \cdots \pi$ interactions. Goddard et al. (1995) correlated the short value for the $b$ axis to the increased flattening of the herringbone pattern as a consequence of the ability of close-packing of the compound rather than to the formation of intermolecular $\pi \cdots \pi$ interactions. $\beta$-type structures are highly non-planar molecules: interestingly, benzodicoronene, pyrene and phenanthrene are exceptions to this. The angle between neighbouring molecules is determined by the ability of the columns to close-pack. Goddard et al. (1995) noted that columns with a circular cross-section pack less efficiently than columns with elliptical cross-sections. Molecules with an elliptical shape have a natural tendency to pack efficiently without extensive tilting. The effect of pressure on the crystallization of disk-shaped molecules such as pyrene and phenanthrene is to afford close-packed structures through the flattening of layers, thereby enhancing $\pi \cdots \pi$ contributions, rather than by titling them further. Perhaps the elliptical shape of naphthalene and the resulting efficient packing exhibited in the known modification can tentatively be

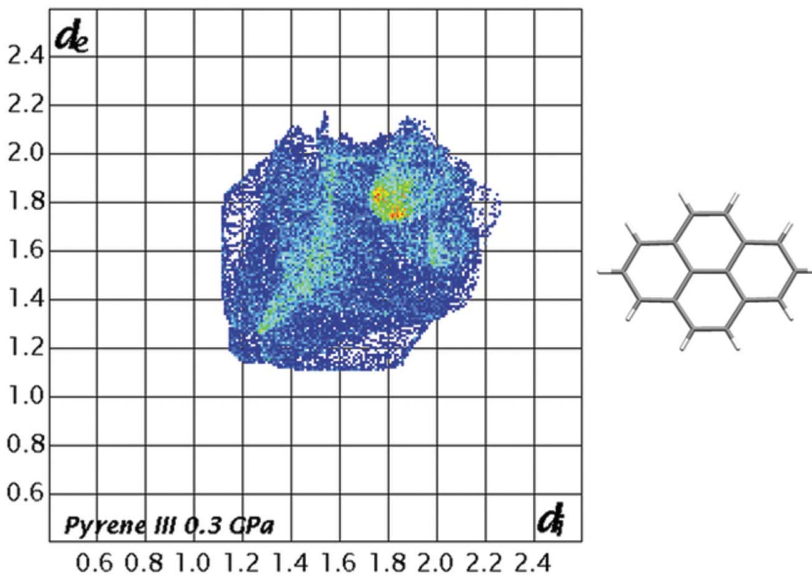

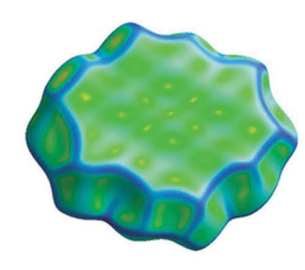

(a)

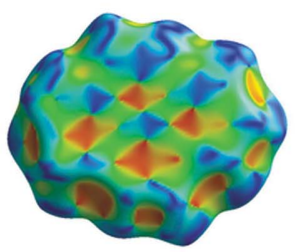

(b)

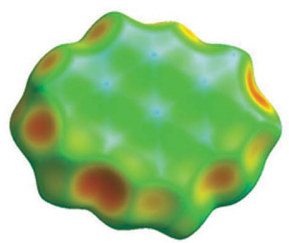

(c)

Figure 13

Hirshfeld surfaces and fingerprint plot for pyrene-(III) at $0.3 \mathrm{GPa}$. The molecule is shown with the Hirshfeld surface mapped with $(a)$ curvedness, $(b)$ shape index and $(c) d_{e}$.

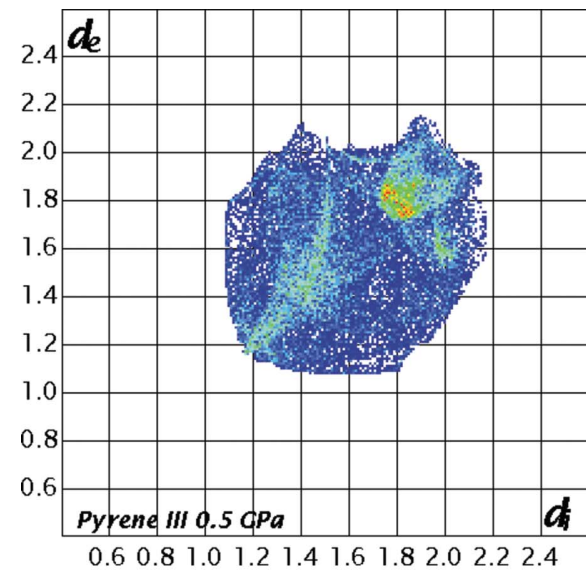

(a)

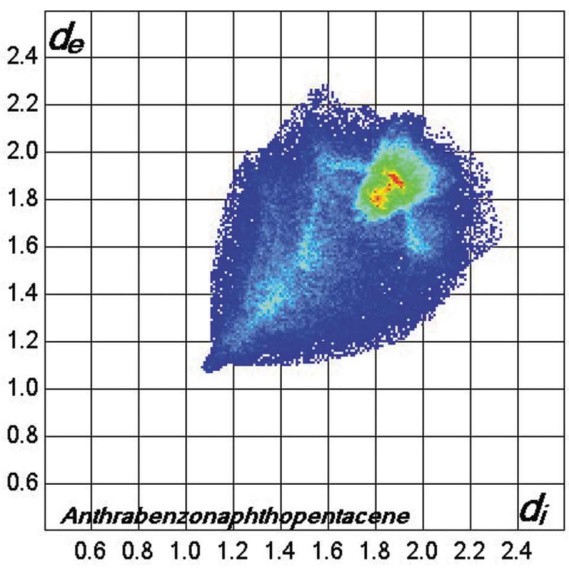

(b)

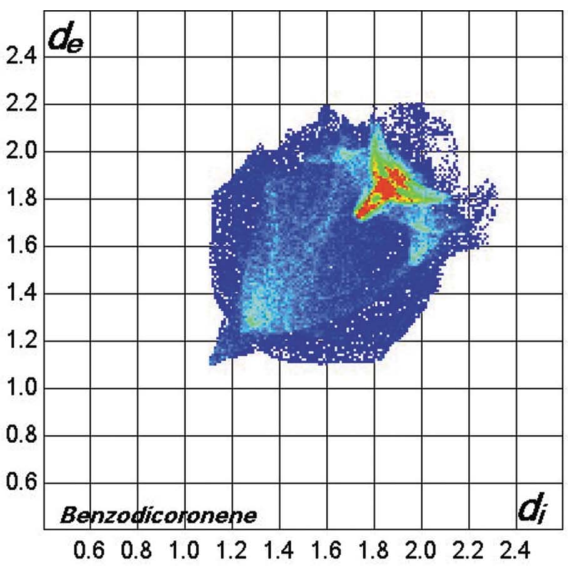

(c)

Figure 14

Fingerprint plots for $(a)$ pyrene-(III) at $0.5 \mathrm{GPa},(b)$ anthrabenzonaphthopentacene (CSD reference code BOXGAW01) and $(c)$ benzodicoronene $(\mathrm{CSD}$ reference code YOFCUR). 
included in the reasons why a new polymorph has not yet been found for this molecule.

Another example where an ambient-pressure structure shows features typical of more than one structure type on analysis of fingerprint plots and Hirshfeld surfaces is given by diperinaphthyleneanthracene (NAPANT01), which is classified as a $\beta$-type structure by Desiraju and Gavezzotti, but its corresponding fingerprinting plot shows $\pi \cdots \pi$ stacking as well as $\mathrm{C}-\mathrm{H} \cdots \pi$ contacts (McKinnon et al., 2004). This 'anomaly' is supported by the fact that the value of its shortest cell axis [7.803 (1) $\AA$ ] is larger than usually found for $\beta$-type structures.

\section{Conclusions}

Recrystallization of naphthalene in the 0.2-0.6 GPa pressure range does not result in the formation of a new polymorph. Furthermore, direct compression of a single crystal to $2.1 \mathrm{GPa}$ does not result in a phase transition.

New polymorphs of pyrene and phenanthrene [denoted pyrene-(III) and phenanthrene-(III), respectively] have been obtained by recrystallization from dichloromethane solution at elevated pressures. Investigation of the topology of molecular centroids, and in particular of Hirshfeld surfaces and fingerprint plots, shows that the mode of packing of these high-pressure polymorphs is significantly different from the ambient-pressure forms in which $\mathrm{C}-\mathrm{H} \cdots \pi$ contacts play a major role. The high-pressure forms are dominated by $\pi \cdots \pi$ contacts with only a small contribution from $\mathrm{C}-\mathrm{H} \cdots \pi$ interactions. These structures deviate from the geometrical model proposed by Desiraju and Gavezzotti that classified PAHs on the basis of the interplanar angle between neighbouring molecules and the length of the shortest crystallographic axis. From our results, it is suggested that $\pi$-stacking becomes more important for high-pressure structures and that the length of the shortest crystallographic axis determines their classification. It is anticipated that the new high-pressure polymorphs of other PAHs may also deviate from the Desiraju-Gavezzotti model and studies are currently underway to explore this area.

One of the authors (FPAF) would like to thank the Leverhulme Trust for funding of a Postdoctoral Fellowship. We also thank Dr B. Dittrich (University of Western Australia, Australia) for helpful discussions and critical reading of the manuscript, Dr J. J. McKinnon (University of Western Australia, Australia) for his help with CRYSTAL EXPLORER and Professor V. Blatov (Samara University, Russia) for his advice on the use of TOPOS.

\section{References}

Abrahams, S. C., Robertson, J. M. \& White, J. G. (1949). Acta Cryst. 2 , 233-244.

Allmann, R. (1970). Z. Kristallogr. 132, 129-151.

Alt, H. C. \& Kalus, J. (1982). Acta Cryst. B38, 2595-2600.

Angel, R. J., Bismayer, U. \& Marshall, W. G. (2001). J. Phys. Condens. Matter, 13, 5353-5364.

Basak, B. S. (1950). Indian J. Phys. 24, 309-314.
Bernstein, J., Davis, R. E., Shimoni, L. \& Chang, N.-L. (1995). Angew. Chem. Int. Ed. Engl. 34, 1555-1573.

Blatov, V. A., Shevchenko, A. P. \& Serezhkin, V. N. (2000). J. Appl. Cryst. 33, 1193.

Blessing, R. H. (1995). Acta Cryst. A51, 33-38.

Block, S., Weir, C. E. \& Piermarini, G. J. (1970). Science, 169, 586-587.

Boldyreva, E. V. (2004a). J. Mol. Struct. 700, 151-155.

Boldyreva, E. V. (2004b). High Pressure Crystallography, NATO Science Series II Mathematics, Physics \& Chemistry, edited by A. Katrusiak \& P. F. McMillan, pp. 495-512. Dordrecht, The Netherlands: Kluwer Academic Publishers.

Boldyreva, E. V., Shakhtshneider, T. P., Sowa, H. \& Uchtmann, H. (2002). J. Thermal Anal. Calorim. 68, 437-452.

Botoshansky, M., Herbstein, F. H. \& Kapon, M. (2003). Helv. Chim. Acta, 86, 113-1128.

Bridgman, P. W. (1938). Proc. Am. Acad. Arts. Sci. 72, 227-268.

Brock, C. P. \& Dunitz, J. D. (1982). Acta Cryst. B38, 2218-2228.

Bruker AXS Inc. (2003). SAINT, Version 7.01A. Bruker AXS Inc., Madison, Wisconsin. USA.

Bruno, I. J., Cole, J. C., Edgington, P. R., Kessler, M., Macrae, C. F., McCabe, P., Pearson, J. \& Taylor, R. (2002). Acta Cryst. B58, 389397.

Camerman, A. \& Trotter, J. (1965). Acta Cryst. 18, 636-643.

Cruickshank, D. W. J. (1957). Acta Cryst. 10, 504-508.

Crystal Impact (2004). DIAMOND, Version 3.0. Crystal Impact GbR, Postfach 1251, 53002 Bonn, Germany. http://www.crystalimpact.com/diamond.

Davydov, V. A., Kashevarova, L. S. \& Revin, O. G. (1996). Russ. J. Phys. Chem. 70, 1012-1015.

Dawson, A., Allan, D. R., Belmonte, S. A., Clark, S. J., David, W. I. F., McGregor, P. A., Parsons, S., Pulham, C. R. \& Sawyer, L. (2005). Cryst. Growth Des. pp. 1415-1427.

Dawson, A., Allan, D. R., Parsons, S. \& Ruf, M. (2004). J. Appl. Cryst. 37, 410-416.

Desiraju, G. R. \& Gavezzotti, A. (1989a). J. Chem. Soc. Chem. Commun. pp. 621-623.

Desiraju, G. R. \& Gavezzotti, A. (1989b). Acta Cryst. B45, 473-482.

Dunitz, J. D. \& Bernstein, J. (1995). Acc. Chem. Res. 28, 193-200.

Etter, M. C., MacDonald, J. C. \& Bernstein, J. (1990). Acta Cryst. B46, 256-262.

Farrugia, L. J. (1999). J. Appl. Cryst. 32, 837-838.

Fabbiani, F. P. A., Allan, D. R., David, W. I. F., Dawson, A. D., McGregor, P. A., Oswald, I. D. H., Parsons, S. \& Pulham, C. R. (2003). Chem. Commun. pp. 3004-3005.

Fabbiani, F. P. A., Allan, D. R., David, W. I. F., Moggach, S. A., Parsons, S. \& Pulham, C. R. (2004). CrystEngComm, 6, 504-511.

Fabbiani, F. P. A., Allan, D. R., Marshall, W. G., Parsons, S., Pulham, C. R. \& Smith, R. I. (2005). J. Cryst. Growth, 275, 185-192.

Fabbiani, F. P. A., Allan, D. R., Parsons, S. \& Pulham, C. R. (2005). CrystEngComm, 7, 179-186.

Frampton, C. S., Knight, K. S., Shankland, N. \& Shankland, K. (2000). J. Mol. Struct. 520, 29-32.

Goddard, R., Haenel, M. W., Herndon, W. C., Kruger, C. \& Zander, M. (1995). J. Am. Chem. Soc. 117, 30-41.

Gonikberg, M. G., Shakhovskoi, G. P. \& Petrov, A. A. (1966). Russ. J. Phys. Chem 40, 1345-1348.

Goworek, T., Wawryszczuk, J. \& Zaleski, R. (2004). Chem. Phys. Lett. 387, 433-435.

Hamann, S. D. (1978). High Temp. High Press. 10, 503-510.

Harvey, R. G. (1991). Polycyclic Aromatic Hydrocarbons: Chemistry and Carcinogenicity. Cambridge, England: Cambridge University Press.

Hazell, A. C., Larsen, F. K. \& Lehmann, M. S. (1972). Acta Cryst. B28, 2977-2984.

Hazen, R. M. \& Finger, L. W. (1982). Comparative Crystal Chemistry, p 811. Chichester: John Wiley and Sons.

Hemley, R. J. \& Dera, P. (2000). Rev. Min. Geochem. 41, 335-419.

Hervey, P. R. \& Foise, J. W. (2001). Miner. Metall. Proc. 18, 1-4. 
Jones, P. F. \& Nicol, M. (1968). J. Chem. Phys. 48, 5440-5448.

Jones, W. \& Cohen, M. D. (1977). Mol. Cryst. Liq. Cryst. 41, 103-107.

Jones, W., Ramdas, S. \& Thomas, J. M. (1978). Chem. Phys. Lett. 54, 490-493.

Kai, Y., Hama, F., Yasuoka, N. \& Kasai, N. (1978). Acta Cryst. B34, 1263-1270.

Kay, M. I., Okaya, Y. \& Cox, D. E. (1971). Acta Cryst. B27, 26-33.

King, H. E. \& Finger, L. W. (1979). J. Appl. Cryst. 12, 374-378.

Knight, K. S. (2005). Private communication.

Knight, K. S., Shankland, K., David, W. I. F., Shankland, N. \& Love, S. W. (1996). Chem. Phys. Lett. 258, 490-494.

Knight, K. S., Shankland, K. \& Shankland, N. (1999). Acta Cryst. A55, Supplement, Abstr. M07.EE.004.

Kroupa, J., Fousek, J., Ivanov, N. R., Březina, B., Pavel, M., Fousková, A., Lhotská, V., Petříček, V. \& Císařová, I. (1988). Solid State Commun. 66, 1002-1006.

Mansour, S. \& Weinreb, A. (1974). Mol. Cryst. Liq. Cryst. 27, 111-125.

McKinnon, J. J., Spackman, M. A. \& Mitchell, A. S. (2004). Acta Cryst. B60, 627-668.

Merrill, L. \& Bassett, W. A. (1974). Rev. Sci. Instrum. 45, 290-294.

Moggach, S., Allan, D. R., Clark, S. J., Gutmann, M. J., Parsons, S., Pulham, C. R. \& Sawyer, L. (2006). Acta Cryst. B62, 296-309.

Moggach, S. A., Allan, D. R., Morrison, C. A., Parsons, S. \& Sawyer, L. (2005). Acta Cryst. B61, 58-68.

Moggach, S. A., Allan, D. R., Parsons, S. \& Sawyer, L. (2006). Acta Cryst. B62, 310-320.

Moggach, S., Allan, D. R., Parsons, S., Sawyer, L. \& Warren, J. E. (2005). J. Synchrotron Rad. 12, 598-607.

Natkaniec, I., Belushkin, A. V., Dyck, W., Fuess, H. \& Zeyen, C. M. E. (1983). Z. Kristallogr. 163, 285-293.

Nicol, M., Vernon, M. \& Woo, J. T. (1975). J. Chem. Phys. 63, 19921999.

Oehzelt, M., Resel, R. \& Nakayama, A. (2002). Phys. Rev. B, 66, 174101/1-174101/5.

Oddershede, J. \& Larsen, S. (2004). J. Phys. Chem. A, 108, 1057-1063.
Parsons, S. (2004). SHADE. The University of Edinburgh, Scotland. Pawley, G. S. \& Yeats, E. A. (1969). Acta Cryst. B25, 2009-2013.

Peresypkina, E. V. \& Blatov, V. A. (2000a). Acta Cryst. B56, 501511.

Peresypkina, E. V. \& Blatov, V. A. (2000b). Acta Cryst. B56, 10351045.

Petříček, V., Císařová, I., Hummel, L., Kroupa, J. \& Březina, B. (1990). Acta Cryst. B46, 830-832.

Piermarini, G. J., Block, S., Barnett, J. D. \& Forman, R. A. (1975). J. Appl. Phys. 46, 2774-2780.

Podsiadło, M., Dziubek, K. \& Katrusiak, A. (2005). Acta Cryst. B61, 595-600.

Ponomarev, V. I., Filipenko, O. S. \& Atovmyan, L. O. (1976). Crystallogr. Rep. 21, 392.

Resel, R., Oehzelt, M., Shimizu, K., Nakayama, A. \& Takemura, K. (2004). Solid State Commun. 129, 103-106.

Robertson, J. M. \& White, J. G. (1947). J. Chem. Soc. pp. 358-368.

Sheldrick, G. M. (1997). SHELXTL, Version 5.10. Bruker AXS Inc., Madison, Wisconsin, USA.

Sheldrick, G. M. (2002). CELL_NOW. University of Göttingen, Germany.

Sheldrick, G. M. (2004). SADABS, Version 2004/1. University of Göttingen, Germany.

Spackman, M. A. \& McKinnon, J. J. (2002). CrystEngComm, 4, 378 392.

Spek, A. L. (2003). PLATON. University of Utrecht, The Netherlands.

Ueberreiter, K. \& Orthmann, H. J. (1950). Z. Naturforsch. Teil A, 5, 101-108.

Vaidya, S. N. \& Kennedy, G. C. (1971). J. Chem. Phys. 55, 987-992.

Wolff, S. K., Grimwood, D., McKinnon, J., Jayatilaka, D. \& Spackman, M. (2005). Crystal Explorer, Version 1.5.1. University of Western Australia, Australia.

Zallen, R., Griffiths, C. H., Slade, M. L., Hayek, M. \& Brafman, O. (1976). Chem. Phys. Lett. 39, 85-89. 